\title{
Frontal Cortical and Subcortical Projections Provide a Basis for Segmenting the Cingulum Bundle: Implications for Neuroimaging and Psychiatric Disorders
}

\author{
Sarah R. Heilbronner and Suzanne N. Haber \\ Department of Pharmacology and Physiology, University of Rochester Medical Center, Rochester, New York 14642
}

The cingulum bundle (CB) is one of the brain's major white matter pathways, linking regions associated with executive function, decision-making, and emotion. Neuroimaging has revealed that abnormalities in particular locations within the $\mathrm{CB}$ are associated with specific psychiatric disorders, including depression and bipolar disorder. However, the fibers using each portion of the CB remain unknown. In this study, we used anatomical tract-tracing in nonhuman primates (Macaca nemestrina, Macaca fascicularis, Macaca mulatta) to examine the organization of specific cingulate, noncingulate frontal, and subcortical pathways through the $\mathrm{CB}$. The goals were as follows: (1) to determine connections that use the $\mathrm{CB}$, (2) to establish through which parts of the CB these fibers travel, and (3) to relate the $\mathrm{CB}$ fiber pathways to the portions of the $\mathrm{CB}$ identified in humans as neurosurgical targets for amelioration of psychiatric disorders. Results indicate that cingulate, noncingulate frontal, and subcortical fibers all travel through the $\mathrm{CB}$ to reach both cingulate and noncingulate targets. However, many brain regions send projections through only part, not all, of the CB. For example, amygdala fibers are not present in the caudal portion of the dorsal CB. These results allow segmentation of the $\mathrm{CB}$ into four unique zones. We identify the specific connections that are abnormal in psychiatric disorders and affected by neurosurgical interventions, such as deep brain stimulation and cingulotomy.

Key words: amygdala; cingulate; deep brain stimulation; diffusion imaging; limbic system; white matter

\section{Introduction}

The cingulum bundle $(\mathrm{CB})$ connects brain regions essential for executive function, decision-making, and emotion processing. It extends through the frontal, parietal, and temporal cortices (Bevore, 1891), connecting the cingulate cortex with proximal and distal limbic structures. Given the extent of the bundle, it is not surprising that volume and integrity changes associated with psychiatric disorders are often found in specific CB subregions ( $\mathrm{Sz}$ eszko et al., 2005; Keedwell et al., 2012). Moreover, distinct CB areas are neurosurgical targets for major depressive disorder (MDD) and obsessive-compulsive disorder (OCD) (Mayberg et al., 2005; Greenberg et al., 2010). Although many connections through the bundle have been characterized, several have not. Furthermore, there is little information concerning where, within the bundle, fibers connecting specific regions travel. Understanding the organization of connections through the $\mathrm{CB}$ is key for mapping the relationship between white matter changes

Received Dec. 19, 2013; revised June 18, 2014; accepted June 19, 2014.

Author contributions: S.R.H. and S.N.H. designed research; S.R.H. and S.N.H. performed research; S.R.H. and S.N.H. analyzed data; S.R.H. and S.N.H. wrote the paper.

This work was supported by National Institute of Mental Health Grants MH 086400 and MH045573. S.N.H. received speaker honorarium from Pfizer and Medtronic.

The authors declare no competing financial interests.

Correspondence should be addressed to Dr. Suzanne N. Haber, Department of Pharmacology and Physiology, University of Rochester School of Medicine and Dentistry, 601 Elmwood Avenue, Rochester, NY 14642. E-mail: Suzanne_haber@urmc.rochester.edu.

DOI:10.1523/JNEUROSCI.5459-13.2014

Copyright $\odot 2014$ the authors $\quad 0270-6474 / 14 / 3410041-14 \$ 15.00 / 0$ associated with a disorder (or treatment) and the connections involved. Thus, the first goal of this study was to characterize cingulate, noncingulate frontal, and specific subcortical pathways through the $\mathrm{CB}$. The second goal was to segment the $\mathrm{CB}$ with respect to which connections pass through each subregion. The third goal was to relate $\mathrm{CB}$ pathways to deep brain stimulation (DBS) and cingulotomy targets for amelioration of psychiatric disorders.

Overall, studies have emphasized the role of the CB in connecting cingulate cortex (Kappers et al., 1936), primarily with other cingulate areas, but also with frontal and parietal regions and the anterior thalamus (Mufson and Pandya, 1984; Morris et al., 1999). However, we know little about whether the CB also contains fibers that neither originate nor terminate in cingulate cortex or where specific connections travel within the bundle. Finally, although we can assume that subcortical projections from specific neurotransmitter systems and amygdala travel through the $\mathrm{CB}$ to reach cingulate cortex, these have not been clearly demonstrated.

Our CB segmentation was designed to provide a guide for interpreting neuroimaging studies. Abnormalities in specific CB subregions highlight the need to establish which connections pass through that subregion. For example, fractional anisotropy is reduced in the anterior CB in bipolar disorder (Wang et al., 2008) and in the subgenual CB in those vulnerable to MDD (Keedwell et al., 2012). Segmentation is also critical for understanding the effects of neurosurgeries that target the CB. Cingulotomy lesions 
for MDD and OCD ablate the rostral dorsal CB (Baer et al., 1995), whereas the subcallosal $\mathrm{CB}$ is the DBS target for MDD (Mayberg et al., 2005). Delineating what goes through each area is critical for linking clinical outcomes with specific target location.

Human connectivity maps depend on anatomical data from animal studies (Croxson et al., 2005). Previously, we demonstrated the organization of monkey ventral prefrontal fibers, then showed that these rules apply in humans (Lehman et al., 2011; Jbabdi et al., 2013). Here, we identified CB fibers from specific cingulate, noncingulate frontal, and subcortical regions in monkeys. We then segmented the bundle into unique zones based on which connections use each part of the CB.

\section{Materials and Methods}

Overview. We injected anterograde or bidirectional tracers into specific brain regions in macaques. Separately, tissue from different animals was processed for tyrosine hydroxylase (TH), serotonin transporter (SERT), or acetylcholinesterase (AChE). Fiber bundles into and through the $\mathrm{CB}$, as well as resulting terminations, were charted for tracer injection cases, as well as SERT, TH, and AChE tissue.

Injection sites. We placed 92 injections of bidirectional and anterograde tracers in specific cortical and subcortical locations. After injections with contamination (in which the tracer was not limited to a single cortical region but had leaked into an adjacent area or into nearby white matter) and weak labeling were eliminated, 54 were chosen for analysis for this study (Figs. 1 and 2). To examine cingulate projections through the $\mathrm{CB}$, there were 8 injections in the dorsal anterior cingulate cortex (dACC, area 24), 4 in the posterior cingulate cortex (PCC, areas 23 and 31 ), and 3 in subgenual anterior cingulate cortex (sACC, areas 25 and 32). To examine noncingulate frontal projections through the $\mathrm{CB}$, there were 8 injections in the dorsolateral frontal cortex (dlFC, lateral areas 9 , 46, 8, and 6), 9 in dorsomedial frontal cortex ( $\mathrm{dmFC}$, medial areas 9 , $9 / 32,8,6$ ), 3 in medial orbitofrontal cortex (mOFC, medial to the medial orbital sulcus, excluding cingulate regions: areas 14, medial 11, medial 13a), 6 in central orbitofrontal cortex (cOFC, between the medial and lateral orbital sulci: areas 13M, 13L, lateral 11, lateral 13a), 2 in lateral OFC (1OFC, lateral to the lateral orbital sulcus: orbital proisocortex, and area $47 / 12$ on the orbital surface), 5 in ventral lateral prefrontal cortex (vlPFC, areas 44, 47, 45), and 2 in the frontal pole (FP, area 10). One animal received three different tracer injections in sACC, IOFC, and cOFC. In addition, to illustrate the location of the subgenual CB (see Fig. $4 C$ ), we used an injection within the subgenual white matter. Finally, to examine the primary subcortical projections through the $\mathrm{CB}$, there were 3 injections in the basolateral amygdala and 1 in the anterior ventral $(\mathrm{AV})$ /lateral dorsal (LD) nucleus of the thalamus. Tissue was also processed for TH, SERT, and AChE to evaluate dopaminergic/noradrenergic, serotonergic, and cholinergic projections through the bundle, respectively.

Surgery, tissue preparation, and analysis. Experiments were performed in accordance with the Institute of Laboratory Animal Resources Guide for the Care and Use of Laboratory Animals and approved by the University Committee on Animal Resources. Adult male macaque monkeys (Macaca nemestrina, Macaca fascicularis, and Macaca mulatta) were tranquilized by intramuscular injection of ketamine $(10 \mathrm{mg} / \mathrm{kg})$. For a subset of animals, MRI ( 3 tesla) T1 or T2 turbo spin echo scans $(0.5 \mathrm{~mm} \times 0.5$ $\mathrm{mm} \times 1.42 \mathrm{~mm}$ ) were obtained before surgery. For the others, serial electrode penetrations were made to locate the anterior commissure, as described previously (Haber et al., 1993). These images and recordings were used to calculate the anterior-posterior, dorsal-ventral, and medial-lateral coordinates for each tracer injection from stereotaxic zero.

Animals received ketamine $10 \mathrm{mg} / \mathrm{kg}$, diazepam $0.25 \mathrm{mg} / \mathrm{kg}$, and atropine $0.04 \mathrm{mg} / \mathrm{kg}$ intramuscularly in the cage. A surgical plane of anesthesia was maintained by either intravenous injections of pentobarbital (for recordings, initial dose $20 \mathrm{mg} / \mathrm{kg}$ i.v., and maintained as needed) or via $1 \%-3 \%$ isoflurane in $100 \%$ oxygen via vaporizer. Temperature, heart rate, and respiration were monitored throughout the surgery. Monkeys were placed in a Kopf stereotaxic, a midline scalp incision was made, and the muscle and fascia were displaced laterally to expose the skull. A craniotomy $\left(\sim 2-3 \mathrm{~cm}^{2}\right)$ was made over the region of interest, and small dural incisions were made only at injection sites.

Monkeys received an injection of one or more of the following anterograde/bidirectional tracers: Lucifer yellow (LY), fluororuby (FR), or fluorescein conjugated to dextran amine (FS) $(40-50 \mathrm{nl}, 10 \%$ in $0.1 \mathrm{M}$ phosphate buffer [PB], pH 7.4; Invitrogen) or tritiated amino acids (100 $\mathrm{nl}, 1: 1$ solution of $\left[{ }^{3} \mathrm{H}\right]$ leucine and $\left[{ }^{3} \mathrm{H}\right]$-proline in $\mathrm{dH}_{2} \mathrm{O}, 200 \mathrm{mCi} / \mathrm{ml}$, NEN). Tracers were pressure-injected over 10 min using a $0.5 \mu \mathrm{l} \mathrm{Ham}$ ilton syringe. After each injection, the syringe remained in situ for 20-30 min. Twelve to $14 \mathrm{~d}$ after surgery, monkeys were again deeply anesthetized and perfused with saline followed by a $4 \%$ PA/1.5\% sucrose solution in $0.1 \mathrm{M} \mathrm{PB}, \mathrm{pH}$ 7.4. Brains were postfixed overnight and cryoprotected in increasing gradients of sucrose $(10 \%, 20 \%$, and $30 \%)$. Serial sections of $50 \mu \mathrm{m}$ were cut on a freezing microtome into $0.1 \mathrm{M} \mathrm{PB}$ or cryoprotectant solution, as described previously (Haber et al., 2006).

One in eight sections was processed free-floating for immunocytochemistry to visualize the tracers. Tissue was incubated in primary anti-LY (1:3000 dilution; Invitrogen), anti-FS (1:1000; Invitrogen), or anti-FR (1:1000; Invitrogen) in $10 \%$ NGS and $0.3 \%$ Triton X-100 (Sigma-Aldrich) in PB for 4 nights at $4^{\circ} \mathrm{C}$. After extensive rinsing, the tissue was incubated in biotinylated secondary antibody followed by incubation with the avidin-biotin complex solution (Vectastain ABC kit, Vector Laboratories). Immunoreactivity was visualized using standard DAB procedures. Staining was intensified by incubating the tissue for $5-15 \mathrm{~s}$ in a solution of $0.05 \% \mathrm{DAB}$ tetrahydrochloride, $0.025 \%$ cobalt chloride, $0.02 \%$ nickel ammonium sulfate, and $0.01 \% \mathrm{H}_{2} \mathrm{O}_{2}$. Sections were mounted onto gel-coated slides, dehydrated, defatted in xylene, and coverslipped with Permount. Sections for autoradiography were mounted on chrome-alum gelatin-coated slides and defatted in xylene overnight. Slides were dipped in Kodak NTB2 photographic emulsion and exposed for $4-6$ months at $4^{\circ} \mathrm{C}$ in a light-tight box. The sections were 


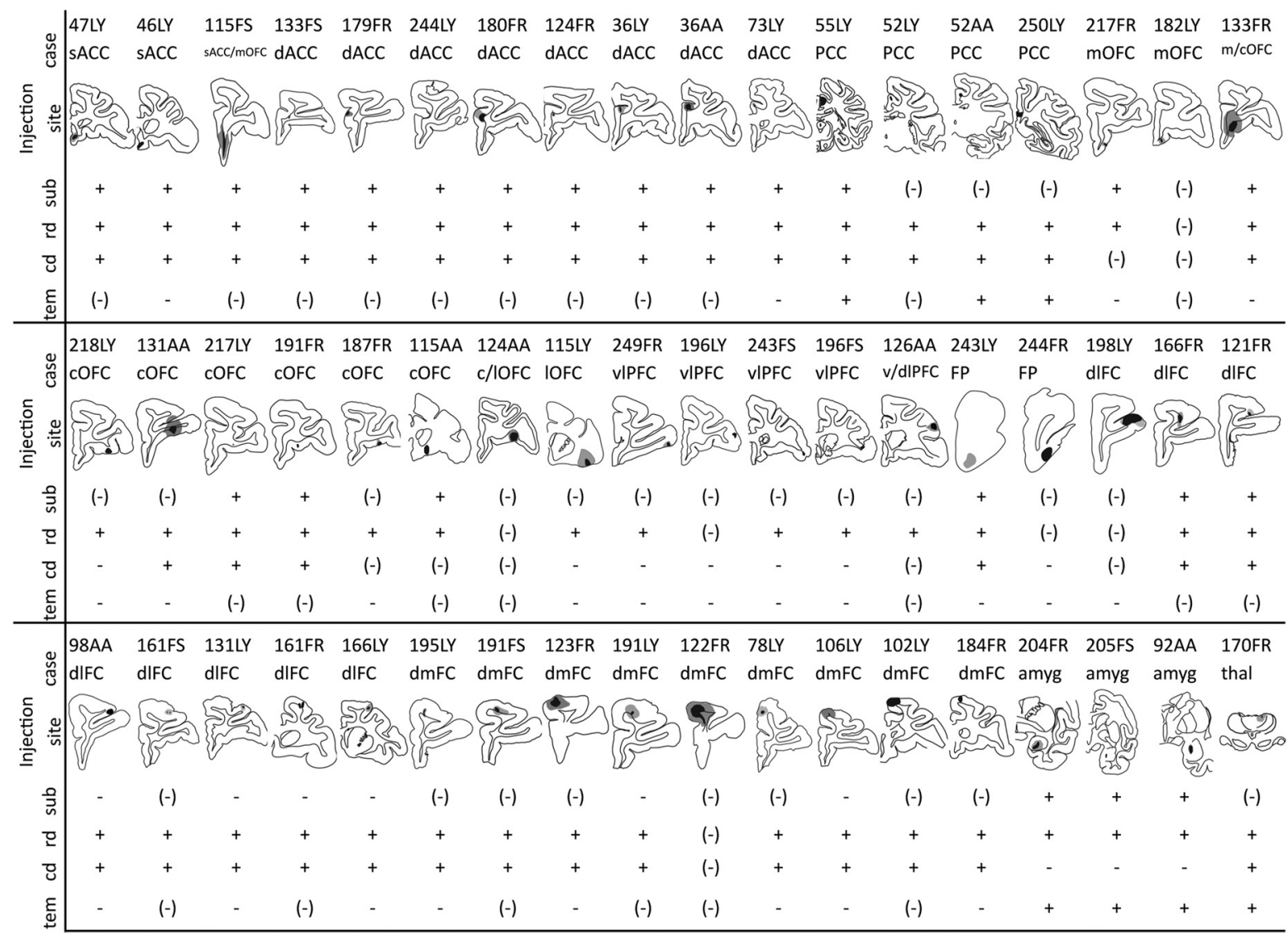

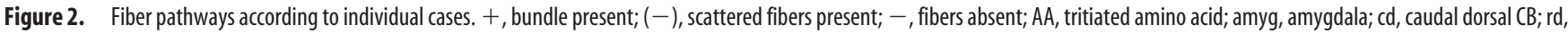
rostral dorsal CB; sub, subgenual CB; tem, temporal CB; thal, thalamus.
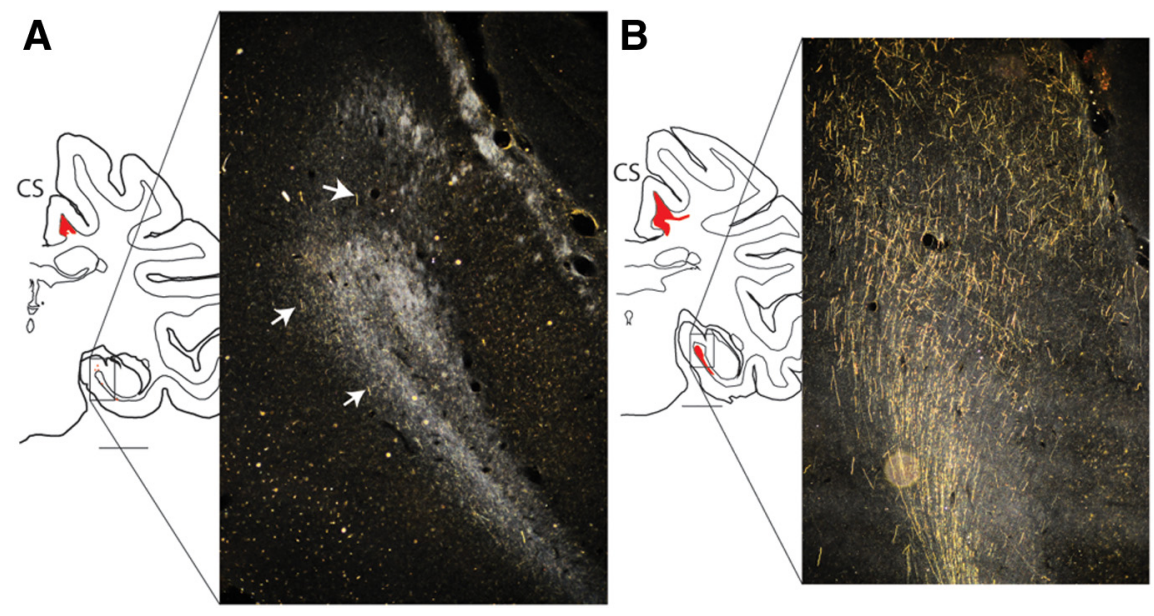

Figure 3. Scattered fibers versus fiber bundles. $\boldsymbol{A}, \mathrm{dlFC}$ scattered fibers (white arrows) in the temporal CB (Case 166FR). Scale bar, $5 \mathrm{~mm}$. B, PCC fiber bundle in the temporal CB (Case 55LY). CS, Cingulate sulcus.

then developed in Kodak D19 for 2.5 min, fixed, washed, and counterstained with cresyl violet.

Monkey tissue processed for TH (Millipore Bioscience Research Reagents; AB152; generated in rabbit) and SERT (Mabtech; ST51-1; generated in mouse) immunocytochemistry was incubated with antisera to TH $(1: 1000)$ or SERT $(1: 150,000)$ in $0.1 \mathrm{M}$ PB with $0.3 \%$ Triton X-100 and $10 \%$ NGS (PBS-T-NGS; Incstar) for four nights at $4^{\circ} \mathrm{C}$ and further processed using the avidin-biotin method (rabbit Elite Vectastain $\mathrm{ABC}$ kit; Vector Laboratories). Sections were rinsed first in PBS, then in Tris buffer $(0.05 \mathrm{M}, \mathrm{pH} 7.6)$, and preincubated in a filtered $0.05 \%$ DAB tetra- hydrochloride solution in Tris buffer for 10 min, before adding $0.01 \% \mathrm{H}_{2} \mathrm{O}_{2}$. Additional tissue was stained with AChE using the Geneser-Jensen/Blackstad method (GeneserJensen and Blackstad, 1971).

Using darkfield light microscopy, brain sections, white matter, fiber bundles, and terminal fields were outlined under a $1.6,4.0$, or $10 \times$ objective with Neurolucida software (MBF Bioscience). Thick fibers without clear terminal boutons were assumed to be passing fibers. Fibers traveling in bundles were outlined as a group. Orientation was indicated for each bundle by charting a few individual fibers within each outline. Labeled fibers were outlined from the injection site, through the $\mathrm{CB}$. We distinguished between bundles of fibers (which could be visualized at low magnification, $1.6 \times$ ) and scattered (few) fibers, and our results are based on the former (for an illustration of these categories, see Fig. 3). To describe efferent axons from each injection site, we carefully followed bundles from their origin through the $\mathrm{CB}$, to their terminal fields. Furthermore, tritiated amino acid injections were used to verify anterograde labeling. Scattered fibers could not always be followed to their terminal fields, so these are not known to be anterograde, and we do not list their destinations.

Cingulotomy lesion. To model the cingulotomy lesion within a macaque brain, we used the anterior-posterior lesion range identified by Steele et al. (2008), extending from MNI $y$-coordinates (rostral-caudal) 2-19. This is not the "typical" lesion but instead represents the entire range used by surgeons across multiple groups. To properly place these 
locations on the smaller monkey brain, we scaled the lesion as well as its distance from the anterior commissure to $40 \%$. This is close to the proportional volume of monkey to human striatum (Yin et al., 2009) as well as the length of the anterior commissure (Lehman et al., 2011). After applying this calculation, we confirmed that landmarks in the human and monkey brains matched at the anterior and posterior ends of the lesion.

Subcallosal DBS electrodes. To model the subcallosal DBS electrodes within a macaque brain, we used the human electrode placement coordinates as described by Hamani et al. (2009). These were scaled based on the proportional distance between the length of the internal capsule from its dorsal boundary with the subcortical WM to the anterior commissure between the monkey and human brain, which is $\sim 40 \%$ and similar to proportional volume of the monkey striatum (Yin et al., 2009). This electrode size and spacing of stimulation sites place each of the sites in approximately the same position in the monkey brain as described in the literature for human placement $(\mathrm{Ha}-$ mani et al., 2009).

\section{Results}

The CB is a long fiber tract that links the frontal, parietal, and temporal lobes (Fig. $4 A$ ). Based broadly on shape and position, the bundle has three basic components: subgenual, dorsal, and temporal. The subgenual portion is positioned ventral to the corpus callosum and lateral to the sACC (Fig. $4 B, C$ ). It lies medial to and merges with the uncinate fasciculus. Although the subgenual CB is narrow, it can be distinguished from the uncinate fasciculus (Fig. $4 C$, inset). Rostrally, it curves around the genu to merge with the dorsal CB (Fig.

$4 B)$. Caudally, it extends until approximately the anterior-posterior level of the rostral border of the putamen. The dorsal portion of the $\mathrm{CB}$ sits lateral to the dorsal cingulate gyrus and ventral to the cingulate sulcus Figure $4 B-E$ ). At its most caudal end, the dorsal CB separates from the cingulate sulcus as the sulcus moves caudally and dorsally to form the marginal ramus (Fig. $4 E$ ). The dorsal CB does not follow the cingulate sulcus dorsally. Instead, it remains in the ventral portion of the white matter, beneath the suprasplenial sulcus. Just behind the splenium (Fig. $4 F$ ), the dorsal $\mathrm{CB}$ merges with the temporal $\mathrm{CB}$, which then extends rostrally into the medial temporal lobe (MTL). It merges with the inferior longitudinal fasciculus and extends rostrally until approximately the midpoint of the lateral geniculate nucleus $(\sim 7.5 \mathrm{~mm}$ anterior to the interaural plane in rhesus macaques) (Schmahmann and Pandya, 2006).

\section{Cortical projection systems \\ Cingulate efferent fibers}

Because cingulate gray matter is adjacent to the white matter of the $\mathrm{CB}$, most cingulate fibers either travel within or simply cross the CB. From the injection site, fibers from all cingulate regions split into a few distinct bundles to reach specific cortical and subcortical targets. Some bundles cross the CB near the injection site to reach subcortical, contralateral, and lateral cortical targets.
Those that enter the CB travel both short and long distances along the anterior-posterior axis to reach other areas of cingulate as well as dmFC, precuneus, MTL, and OFC.

\section{$s A C C$}

As described previously (Lehman et al., 2011), some sACC fibers pass laterally through the subgenual $\mathrm{CB}$ near the injection site and split into bundles, one of which joins the uncinate fasciculus. sACC fibers reach the corpus callosum, superior longitudinal fasciculus, amygdalofugal pathway, internal capsule, external capsule, and extreme capsule. Another set of sACC fibers joins the subgenual CB adjacent to the injection site (Fig. 5A-C). sACC fibers project through the subgenual $\mathrm{CB}$ to reach nearby sACC and mOFC. In addition, they project rostrally and dorsally from the subgenual $\mathrm{CB}$ to reach the dorsal $\mathrm{CB}$ (Fig. $5 B$ ). They travel rostrally and caudally within the dorsal CB to reach dACC, PCC, and retrosplenial cortex (areas 29 and 30) (Fig. 5D). In addition, sACC fibers travel rostrally from both the subgenual and dorsal $\mathrm{CB}$ to enter the anterior uncinate fasciculus and frontopolar white matter, from which they reach FP (anterior medial area 10). Furthermore, some sACC fibers exit the dorsal CB and circumvent the cingulate sulcus to terminate in the $\mathrm{dmFC}$ (areas 8 and 9). Finally, although a small number of sACC fibers do curve around the splenium to terminate in ventral PCC and retrosplenial cortex, they do not extend deep into the temporal lobe. 
A

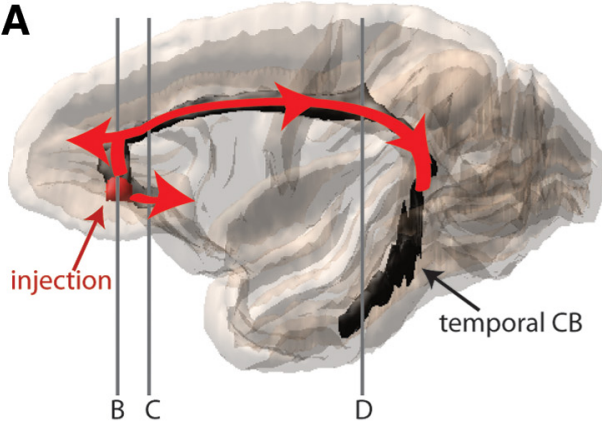

C
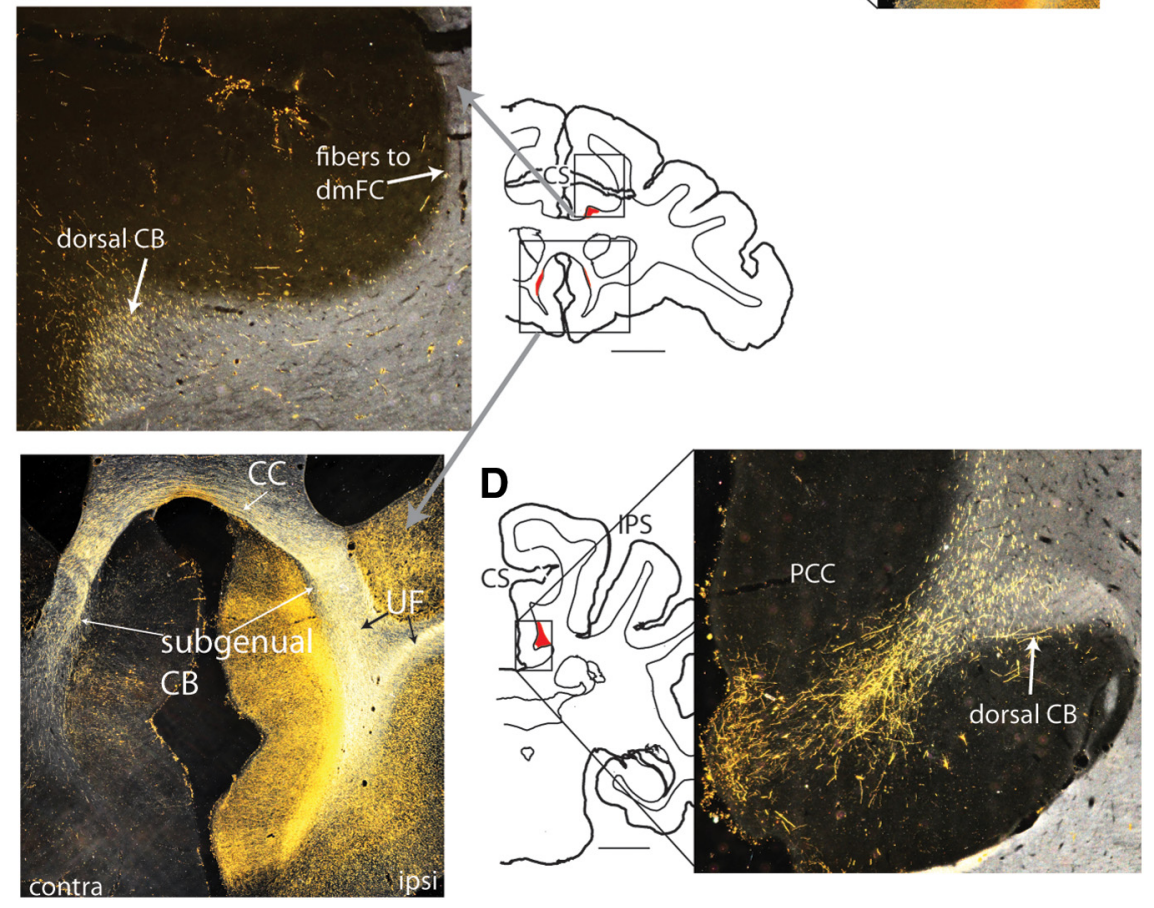

Figure 5. SACC (areas 25 and 32) projections through the (B. Injection shown was between areas 32 and 14 (Case 115FS). $\boldsymbol{A}$, Schematic represents the extent of $\triangle A C C$ projection (illustrated in red) through $C B$. Most fibers do not extend into the temporal $C B$ (indicated in black). B, Fibers travel rostrally and dorsally from the subgenual $(B$ to enter the dorsal CB. C, Bottom, Fibers are particularly dense near the injection site, where they cross the $C B$ and split into bundles (such as the $C(C$ and UF). Top, sACC fibers project through the dorsal $C B$ to reach $\mathrm{AACC}$ and $\mathrm{dmFC} . \mathrm{D}$, sACC fibers project through the dorsal $\mathrm{CB}$ to $\mathrm{PCC}$. CC, Corpus callosum; contra, contralateral; CS, cingulate sulcus; IPS, intraparietal sulcus; ipsi, ipsilateral; UF, uncinate fasciculus.

\section{$d A C C$}

dACC fibers leave the injection site and split immediately into bundles based on their destinations (Fig. 6A-D). dACC capsular, striatal, and callosal fiber bundles cross through the CB laterally and ventrally (Fig. 6B, $C$ ) but do not travel within it. These axons exit in a specific order regardless of the injection site position. The capsular fibers exit the $\mathrm{CB}$ and form a distinct bundle closest to the injection site. Fibers projecting to the striatum and the contralateral hemisphere exit the $\mathrm{CB}$ and form distinct bundles a few millimeters caudal to capsular projections, and travel ventrally to join Muratoff's bundle and the corpus callosum, respectively. In contrast to capsular, striatal and callosal fibers that exit the CB as separate, but discrete bundles, dACC fibers traveling to lateral cortical regions do not form a single bundle, but cross through the $\mathrm{CB}$ for several millimeters as a continuous stream of axons. Together, fibers from a given dACC region that terminate in lateral and contralateral cortical areas, the striatum, thalamus, and brainstem, are no longer within the CB just a few millimeters caudal to the injection site. dACC fibers that do join the dorsal CB travel rostrally and caudally for significant distances to terminate in other dACC areas, the PCC, and retrosplenial cortex (Fig. 6E,F). dACC fibers also project ventrally within the subgenual $\mathrm{CB}$ to reach the sACC. Pregenual dACC fibers travel ventrally directly from the injection site to the subgenual CB (Fig. 6D), whereas postgenual dACC fibers first project rostrally within the dorsal $\mathrm{CB}$, then turn ventrally to reach the subgenual CB. From the subgenual $\mathrm{CB}$, dACC fibers terminate in sACC.

Importantly, the CB links fibers from dACC to noncingulate cortical regions. Like sACC fibers, they travel rostrally from the edge of the $\mathrm{CB}$ to reach FP. In addition, the dorsal $\mathrm{CB}$ carries axons to large regions of dorsomedial cortex. These fibers exit the bundle and circumvent the cingulate sulcus to terminate in medial areas 6,8 , and 9 . These fibers do not seem to terminate again in noncingulate areas until they reach the caudal portion of the dorsal CB, at which point they travel dorsally to reach precuneus. In addition, some dACC axons traveling in the subgenual $\mathrm{CB}$ merge with the uncinate fasciculus to project laterally, terminating in the OFC. Finally, dACC fibers do not appear to travel in main part of the temporal CB.

\section{PCC}

As with dACC and sACC fibers, PCC fibers (Fig. 7A) split into three bundles near the injection site: capsular, striatal, and contralateral cortical. PCC fibers that travel within dorsal $\mathrm{CB}$ terminate in other PCC regions and in the dACC (Fig. 7B). Rostrally, fibers curve ventrally around the genu to reach the subgenual CB to terminate in sACC.

Similar to dACC fibers, PCC fibers also use the dorsal $\mathrm{CB}$ to reach noncingulate frontal regions. However, in addition to $\mathrm{dmFC}$ areas 6 and 9 (Fig. $7 B$; we do not see PCC labeling in area 8 ) and OFC, they also terminate laterally in the dlFC (areas 46 and 9). In contrast to fibers from either the sACC or dACC, PCC fibers do travel in the temporal CB. Axons exit the PCC and project caudally and ventrally around the splenium to reach this part of the CB. These axons terminate in the MTL, most prominently the subiculum (Fig. 7C).

\section{Summary of cingulate fibers}

Cingulate fibers form distinct striatal, callosal, and capsular bundles that cross through the CB near the injection site to reach their targets. In addition, fibers traveling to lateral cortical regions exit the $\mathrm{CB}$ for several millimeters rostral and caudal to the injection site. Thus, any given region of the $\mathrm{CB}$ contains sets of fibers from adjacent cingulate cortex projecting to subcortical, contralateral, and lateral cortical targets. These are fairly specific to nearby cingulate because these fibers have crossed over and exited the $\mathrm{CB}$ within a few millimeters of the injection site. 
A
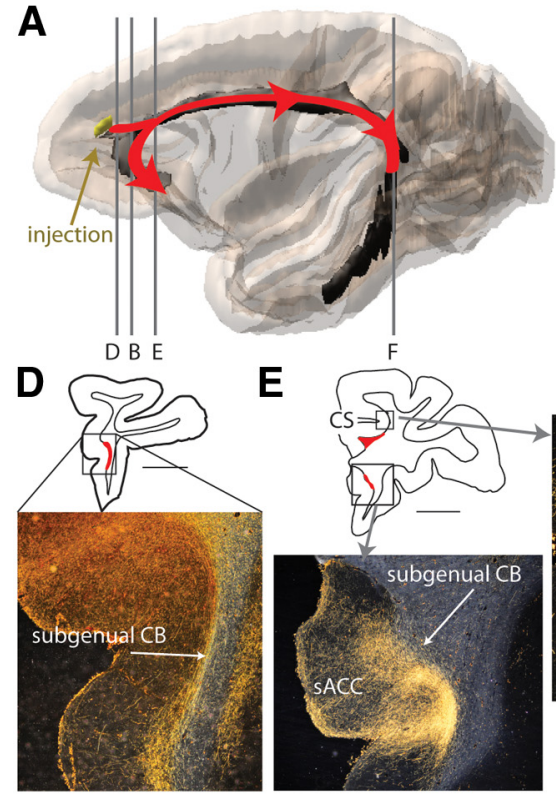

B

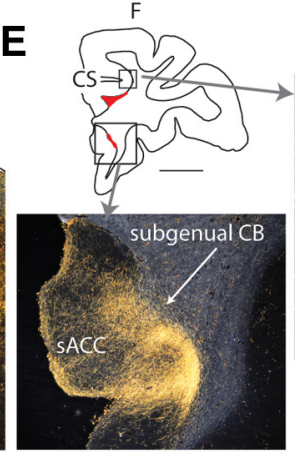

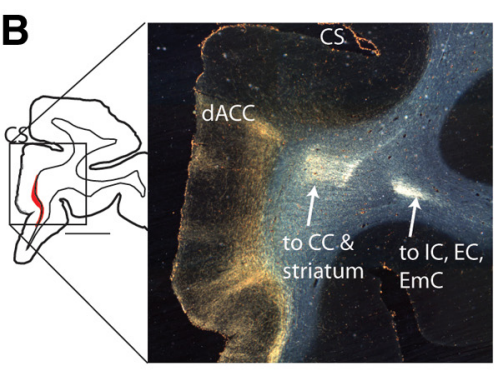

$\mathbf{F}$

$\mathbf{F}$
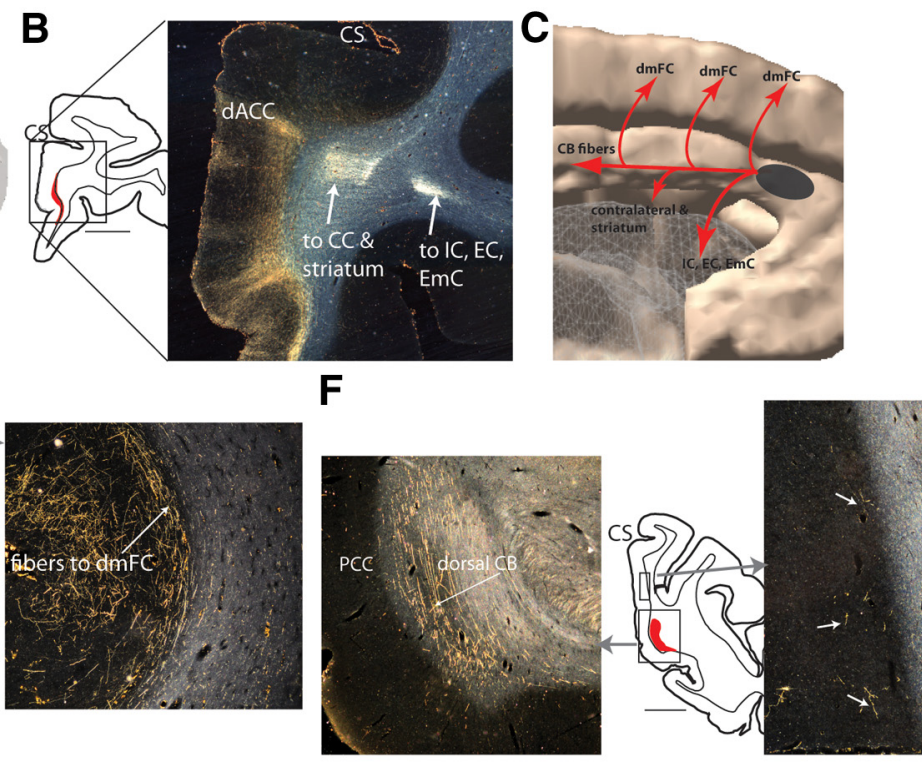

Figure 6. $d A C C$ fibers use the $C B$ (Case 179FR). $A$, Schematic represents the extent of dACC projection (illustrated in red) through CB. Fibers do not extend into the temporal CB (indicated in black). $B$, dACC subcortical and callosal fibers exit the CB near the injection site and split into bundles. $C$, Diagram indicates the order in which dACC fibers cross over the CB. $\boldsymbol{D}$, dACC fibers travel ventrally from the dorsal $C B$ to the subgenual $C B$. $E$, dACC fibers use the dorsal and subgenual $C B$ to terminate in $\mathrm{AACC}, \mathrm{SACC}$, and dorsomedial cortex. $F$, dACC fibers extend to the caudal edge of the dorsal $C B$ to reach $\mathrm{PCC}$ and precuneus (white arrows). CC, Corpus callosum; CS, cingulate sulcus; EC, external capsule; EmC, extreme capsule; IC, internal capsule.
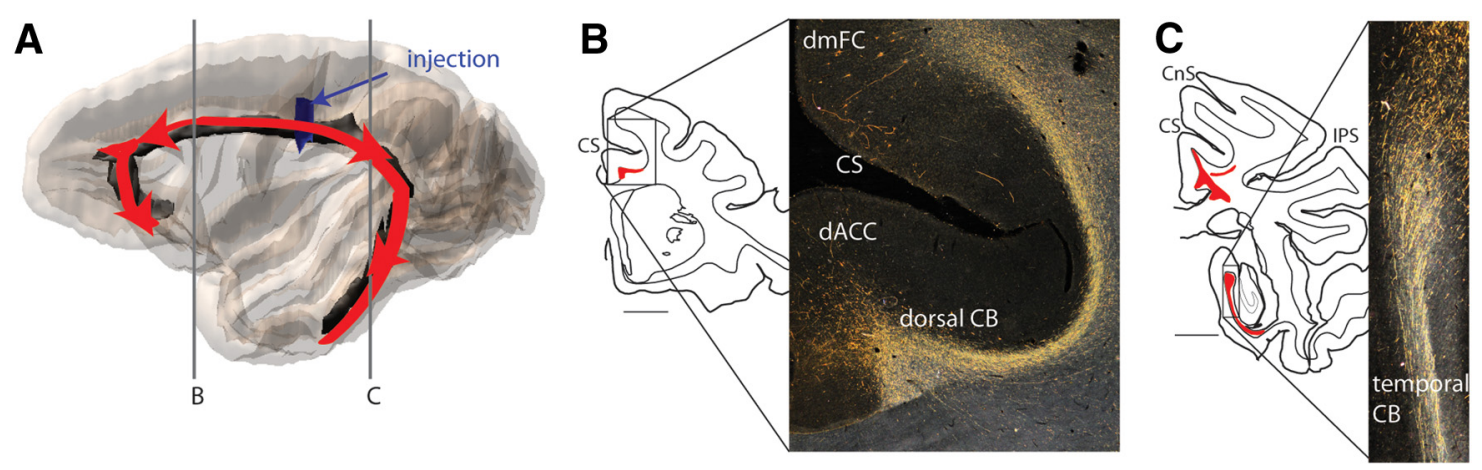

Figure 7. $\mathrm{PCC}$ fibers project through the $\mathrm{CB}$ (Case 55LY). $\boldsymbol{A}$, Schematic represents the extent of $\mathrm{PCC}$ projection through $\mathrm{CB}$ (illustrated in red). $\boldsymbol{B}$, $\mathrm{PCC}$ fibers use the dorsal $C B$ to reach cingulate and dorsomedial cortex. C, PCC fibers, alone among cingulate regions examined, project strongly through the temporal CB. CnS, Central sulcus; CS, cingulate sulcus; IPS, intraparietal sulcus.

As expected, cingulate fibers travel extensively within the $\mathrm{CB}$ to reach other areas of cingulate cortex. However, cingulate fibers also use the $\mathrm{CB}$ to reach noncingulate areas, including $\mathrm{FP}, \mathrm{dmFC}$, OFC, dlFC, and MTL. Many of these axons travel long distances within the $\mathrm{CB}$ before reaching their targets.

\section{Noncingulate frontal cortical efferent fibers \\ OFC}

Similar to sACC fibers, mOFC and cOFC axons (Fig. 8) join the subgenual CB by traveling dorsally and medially from the injection site (Lehman et al., 2011). Many of these axons terminate locally in the sACC. Others continue dorsally around the genu to enter the dorsal CB. These axons travel rostrally and caudally the entire length of the dorsal CB to terminate in AACC, PCC, and retrosplenial cortex. They do not continue into the temporal CB. However, they do terminate in noncingulate regions, including $\mathrm{FP}, \mathrm{dmFC}$, and precuneus.

In contrast, lOFC fibers do not travel the entire length of the dorsal CB, nor are they present in the subgenual or temporal CB. Indeed, we found little evidence that rostral lOFC fibers travel within the CB. However, orbital proisocortex fibers (Fig. 8) do use the $\mathrm{CB}$. These axons travel dorsomedially from the injection site to reach the dorsal CB. They project through the dorsal CB to reach nearby dACC and dmFC (areas 9 and 8 only) but do not extend caudally beyond the level of the anterior commissure.

Dorsal and lateral frontal cortex

Fibers from the diFC travel ventrally and medially from the injection site to join the $\mathrm{CB}$ (Fig. 9A). From there, some fibers travel rostrally and ventrally to reach the subgenual $\mathrm{CB}$, to terminate in sACC. dIFC fibers also travel rostrally and caudally within the dorsal CB to terminate in dACC (Fig. 9B), PCC (Fig. 9C), and retrosplenial cortex. A few dlFC axons project around the splenium to reach the ventral PCC and prosubiculum. However, these fibers do not continue densely into the ventral parts of the temporal CB.

dlFC fibers also use the dorsal CB to reach noncingulate cortical areas. They travel rostrally to reach FP. Interestingly, unlike the $\mathrm{OFC}$ and cingulate fibers described above, they do not use the $\mathrm{CB}$ to access $\mathrm{dmFC}$ (areas 9, 8, 6). Instead, consistent with prior 

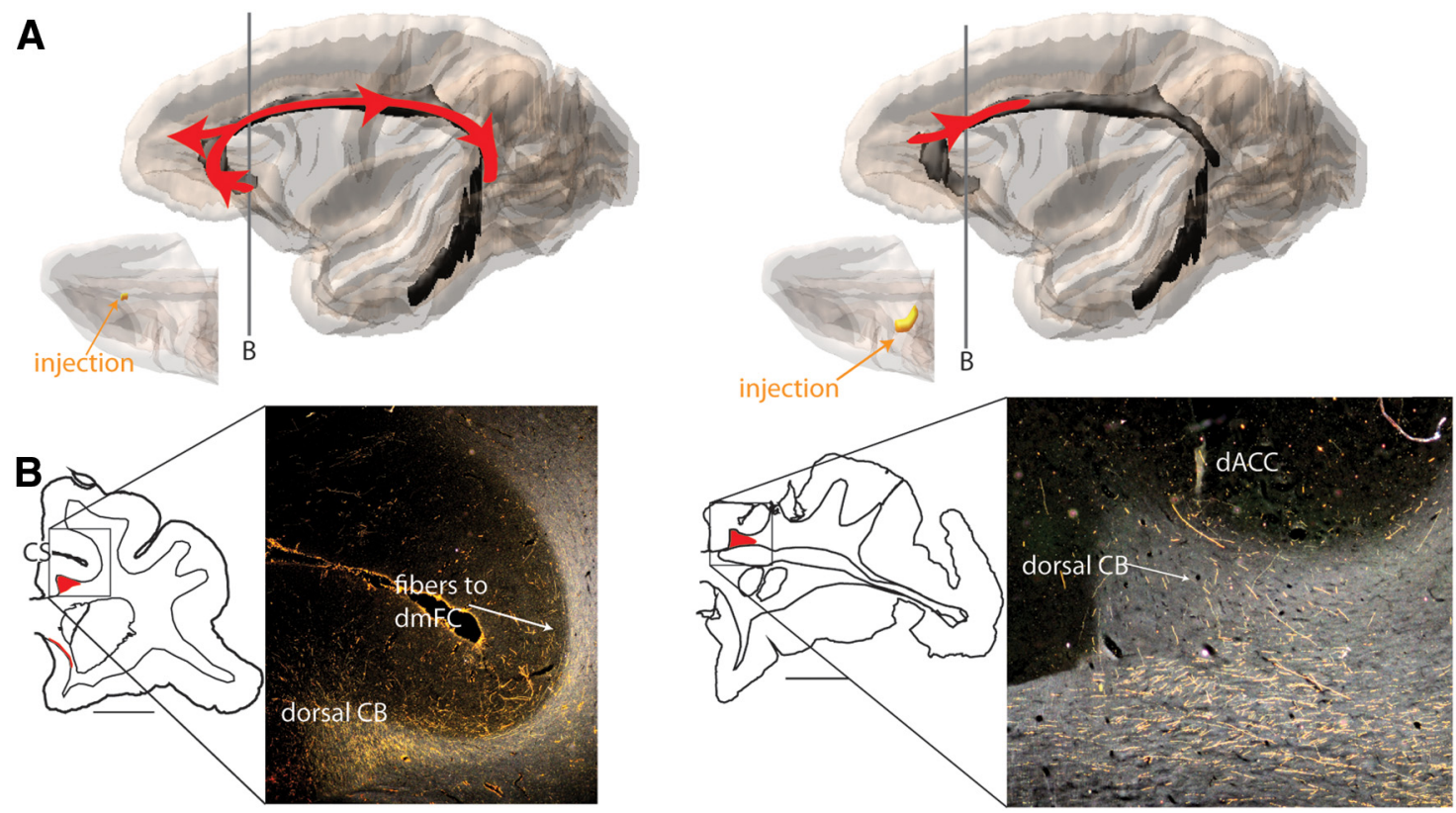

Figure 8. OFC fibers project through the CB. Left, Representative COFC injection (Case 191FR). Right, Representative IOFC (orbital proisocortex) injection (Case 115LY). $\boldsymbol{A}$, Schematics illustrate extent of $\mathrm{COFC}$ and IOFC projections through CB. Red represents $\mathrm{OFC}$ projections; black represents entirety of CB. Insets to left, Orbital views of the injection sites. $B, C \mathrm{COF}$ and IOFC fibers travel through the dorsal $C B$ to reach $\mathrm{AACC}$ and dorsomedial cortex. COFC fibers travel caudally in the dorsal CB to reach PCC, but IOFC fibers do not. CS, Cingulate sulcus.
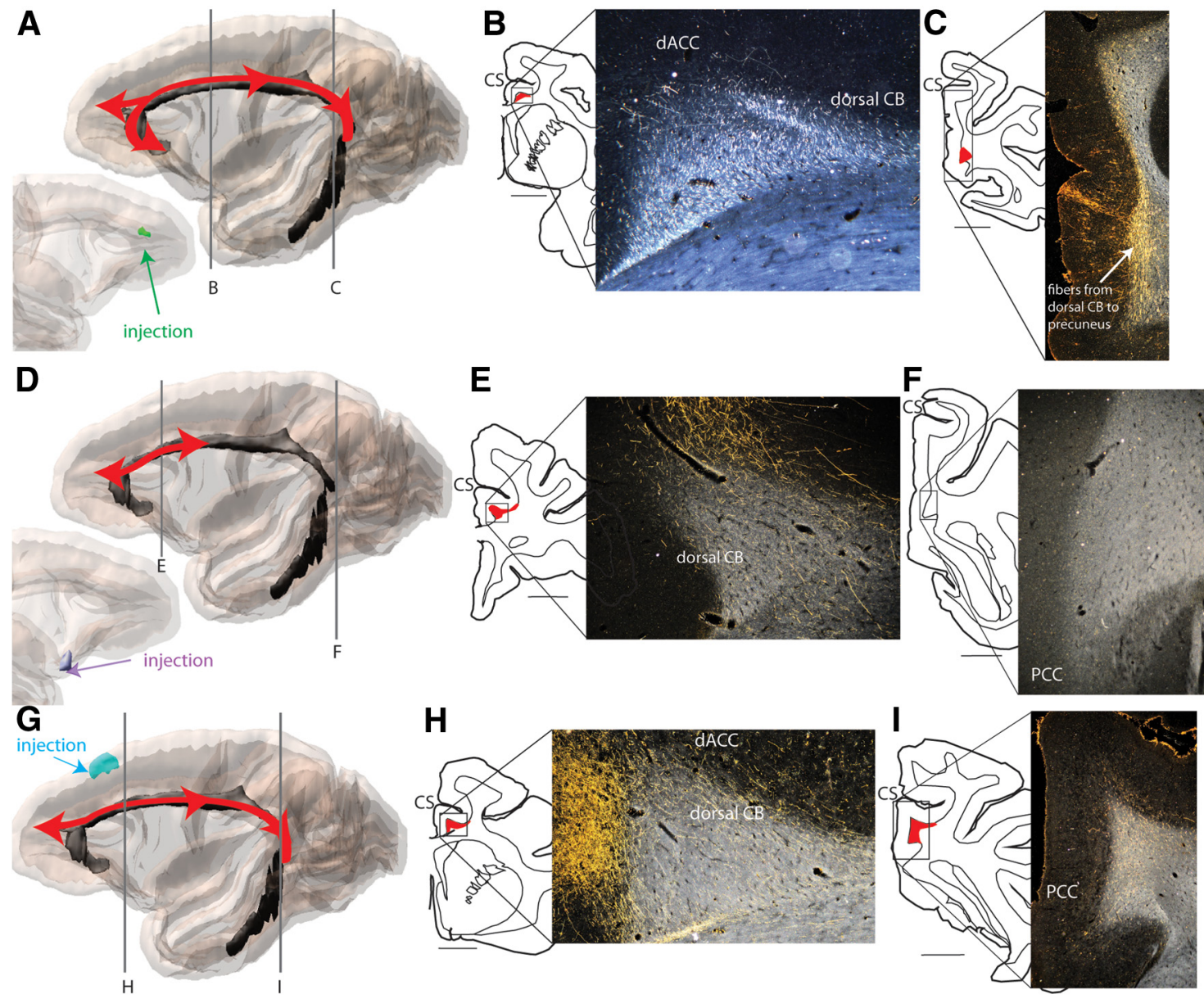

Figure 9. Dorsal and lateral frontal cortical fibers project through the CB. A, Schematic represents the extent of dIFC projection through CB. Red represents dIFC projection; black represents entirety of $(B$. Case shown is 166FR. dIFC fibers project through the dorsal CB to reach $\mathrm{dACC}(\boldsymbol{B})$ and $\mathrm{PCC}(\boldsymbol{C})$. In addition, many fibers also project dorsally from the caudal portion of the $C B$ to reach precuneus. $\boldsymbol{D}$, Red represents vIPFC projection; black represents entirety of CB. Case shown is $243 \mathrm{FS}$. vIPFC fibers project through the rostral dorsal CB $(\boldsymbol{E})$ but not the caudal dorsal CB $(\boldsymbol{F})$. $\boldsymbol{G}$, Red represents $\mathrm{dmFC}$ projection; black represents entirety of CB. Case shown is 102LY. $\mathrm{dmFC}$ fibers project through the CB to reach $\mathrm{AACC}(\boldsymbol{H})$ and PCC $(\boldsymbol{I})$. CS, Cingulate sulcus. 
findings (Schmahmann and Pandya, 2006), fibers travel from dlFC to dorsomedial frontal regions directly through the superior longitudinal fasciculus. However, they do travel within the dorsal $\mathrm{CB}$ to reach the precuneus. As the cingulate sulcus moves dorsally at its caudal end, dlFC fibers spread out, covering all of the medial white matter between the cingulate sulcus and the corpus callosum and innervating the precuneus (Fig. 9C).

Fibers from the vlPFC travel dorsally and medially from the injection site to join the dorsal CB (Fig. $9 D-F$ ), without entering the subgenual CB. They use the CB to terminate in AACC and dmFC. However, at approximately the level of the anterior commissure, they end, and most do not appear to extend into the caudal part of the dorsal CB or the temporal CB.

$\mathrm{dmFC}$ fibers travel ventrally and circumvent the cingulate sulcus to join the dorsal $\mathrm{CB}$ and travel rostrally and caudally within the bundle to terminate in AACC, PCC, and retrosplenial cortex (Fig. 9G-I). However, they do not use it to reach other dorsomedial cortical regions. Rostrally, a few dmFC fibers travel ventrally to reach pregenual area 32, but, for the most part, they do not continue subgenually.

\section{Frontal polar cortex}

The projections of FP fibers through the CB have been detailed previously (Petrides and Pandya, 2007). Although there may be variation among subregions (Fig. 2), we do observe FP fibers traveling through the subgenual and dorsal $\mathrm{CB}$ to reach cingulate (sACC, dACC, PCC, and retrosplenial cortex) and dmFC. Few FP fibers extend into the temporal CB.

\section{Summary of noncingulate cortical fibers}

Noncingulate frontal cortical projections through the $\mathrm{CB}$ use the subgenual and dorsal regions of the $\mathrm{CB}$ but do not extend strongly into the temporal $\mathrm{CB}$. Unlike the other frontal fibers, $\mathrm{dmFC}, \mathrm{OFC}$, and vlPFC do not extensively use the subgenual CB. vlPFC and lOFC fibers are mostly absent from the caudal portion of the dorsal CB. Noncingulate frontal fibers use the CB to project to all cingulate regions, including sACC, dACC, PCC, and retrosplenial cortex. However, importantly, these noncingulate cortical fibers also use the $\mathrm{CB}$ to travel to other noncingulate cortical regions, including $\mathrm{FP}, \mathrm{dmFC}$, precuneus, and the prosubiculum. Thus, although the relationship between cingulate cortex and the $\mathrm{CB}$ is strong, the cingulate does not account for the entirety of cortical projections through this bundle.

\section{Contralateral cortical projections within the $\mathrm{CB}$}

Although both cingulate and noncingulate cortical fibers travel within the CB ipsilateral to the side of the injection, it is primarily cingulate fibers that travel in the contralateral $\mathrm{CB}$. They project a short distance to reach contralateral targets near the injection site. Rostral pregenual dACC fibers travel caudally to the corpus callosum and join the contralateral dorsal CB. They travel rostrally within the bundle to innervate contralateral dACC and FP, and ventrally to join the contralateral subgenual CB and terminate in sACC. They do not travel caudally in the contralateral hemisphere (Fig. 10A-C). More caudal dACC fibers do project both rostrally and caudally in the contralateral dorsal CB. sACC fibers project dorsally to reach the corpus callosum and the contralateral subgenual and rostral dorsal CB. PCC fibers cross immediately to the contralateral side, projecting rostrally and caudally a short distance within the caudal portion of the dorsal CB (Fig. 10D). Thus, the majority of the contralateral contribution to the $\mathrm{CB}$
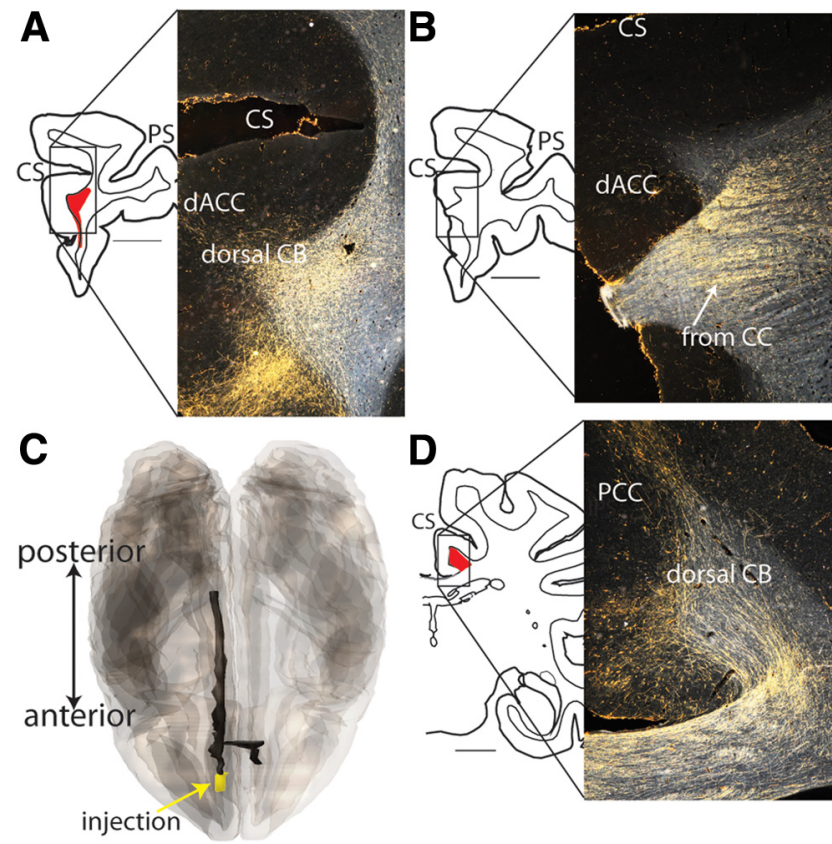

Figure 10. Contralateral projections through the $C B$ are mainly cingulate in origin. $A$, Contralateral pregenual dACC fibers (Case 179FR) travel rostral from the corpus callosum to terminate in dACC. $\boldsymbol{B}$, At the level of the genu of the corpus callosum, pregenual dACC fibers cross to the contralateral side but do not travel caudally within the dorsal CB. C, 3D model (horizontal view) of contralateral rostral $\mathrm{dACC}$ projections. Yellow represents injection site; black represents cingulum bundle fibers. D, Contralateral PCC fibers (Case 55LY) pass through the dorsal CB. CC, Corpus callosum; $C$, cingulate sulcus; $P S$, principal sulcus.

comes from the cingulate itself, and particularly the portion of contralateral cingulate directly opposite any given location within the $\mathrm{CB}$.

\section{Subcortical projection systems through the $\mathrm{CB}$}

Fibers from several key subcortical areas also use the CB to reach their targets. Importantly, these targets are not limited to cingulate cortex. We highlight here the basolateral amygdala; 1 thalamic area; and 3 neurotransmitter systems: catecholamines (dopamine/norepinephrine), serotonin, and acetylcholine.

\section{Basolateral amygdala}

Basolateral amygdala fibers take two routes to reach the CB (Fig. 11). One set of amygdala fibers projects through the ventral amygdalofugal pathway to the subgenual $\mathrm{CB}$ to either terminate in sACC or project rostrally and dorsally to reach the dorsal $\mathrm{CB}$ (Fig. 11B). These dorsal CB fibers terminate in patches along the course of the rostral dACC (Fig. 11C). Importantly, the amygdala uses the $\mathrm{CB}$ to reach noncingulate cortical areas, including $\mathrm{dmFC}$ areas 6,8 , and 9 (Fig. 11C). There were few, if any, amygdala fibers that passed through the dorsal CB caudal to the anterior commissure. Instead, amygdala fibers connect to MTL via a second, caudal route through the temporal CB (Fig. 11D). Interestingly, they do not curve around the splenium to join the dorsal $\mathrm{CB}$. Thus, the caudal part of the dorsal CB does not contain amygdala fibers.

\section{AV/LD thalamus}

Fibers leave the AV/LD and circumvent the ventral and lateral edges of the caudate nucleus. AV/LD fibers project through the internal capsule to join the dorsal $\mathrm{CB}$ as rostral as the anterior commissure and as caudal as the splenium. They then travel dorsally and medially to reach the dorsal portion of the CB (Fig. 
A

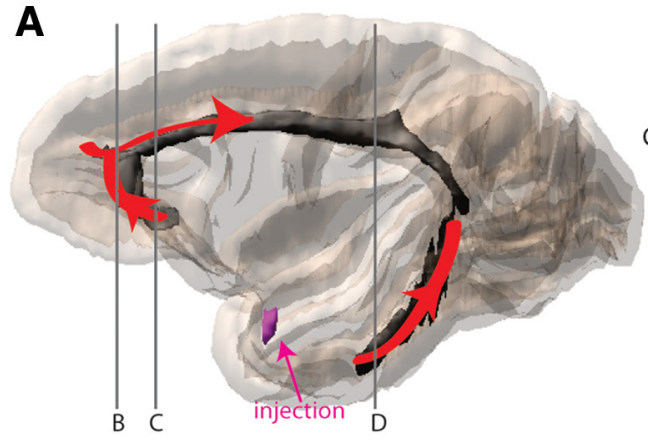

C
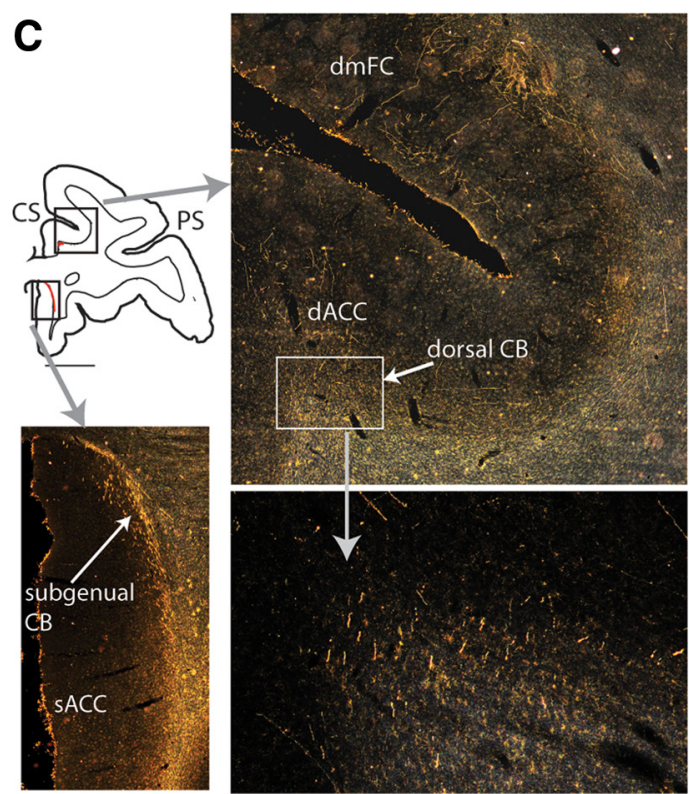

B
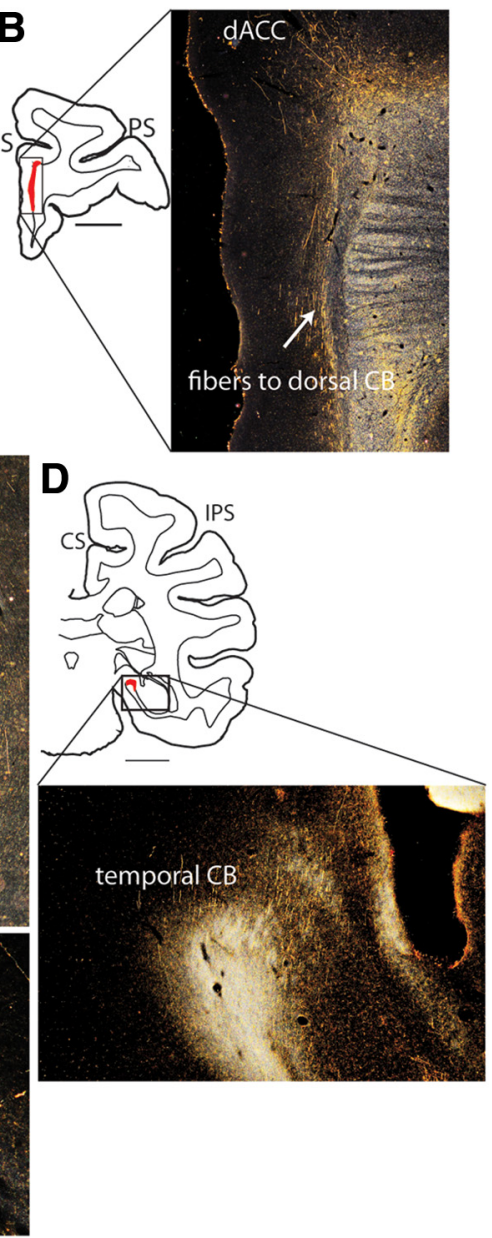

Figure 11. Basolateral amygdala projects through the (B (Case 205FS). A, Schematic represents the extent of amygdala projection through $C B$. Red represents amygdala projection; black represents entirety of $C B . B, C$, One set of amygdala fibers reaches the $C B$ rostrally. These travel through the ventral amygdalofugal pathway to reach the subgenual $C B$, curve dorsally around the genu, and extend caudally into the dorsal CB. They terminate in $\mathrm{AACC}, \mathrm{SACC}$, and dorsomedial cortex. These fibers are no longer present midway through the dorsal $C B$. D, A second set of amygdala fibers reaches the $C B$ caudally. These project through the middle longitudinal fasciculus and inferior longitudinal fasciculus to reach the temporal $C B$. They do not reach the dorsal $C B$, so they do not intersect the rostral set of fibers. CS, Cingulate sulcus; IPS, intraparietal sulcus; PS, principal sulcus.
$12 A-C)$. AV/LD fibers travel in the dorsal $\mathrm{CB}$ to terminate in dACC, PCC, retrosplenial cortex, precuneus, and dmFC. At the splenium, they curve ventrally and rostrally from the dorsal $\mathrm{CB}$ to reach the temporal CB (Fig. 12D) to terminate in the MTL (particularly parasubiculum, presubiculum, and parahippocampal area $\mathrm{TH}$ ). Rostrally, a small number of AV/LD fibers travel ventrally to reach pregenual area 32, but few AV/LD fibers continue ventrally and caudally into the subgenual $\mathrm{CB}$.

\section{Neuromodulator systems}

TH, SERT, and AChE-positive fibers are present throughout the entire CB (Fig. 13A). They take multiple pathways to reach the $\mathrm{CB}$, including, but not limited to, a subgenual route, a temporal route, and, in the case of TH and SERT-positive fibers, a route through the external capsule. TH, SERT, and AChE-positive projections through the CB to the cortex are widespread. TH (Fig. $13 B, C$ ), SERT (Fig. 13D-F), and AChE (Fig. 13G,H) fibers terminate in all areas of cingulate: SACC, dACC, PCC, and retrosplenial cortex. However, they also use the $\mathrm{CB}$ to reach noncingulate areas: FP, dmFC (areas 9, 8, 6), caudal dorsomedial cortex (areas 4, 2, 3, 1, and precuneus), and MTL. SERT and AChE fibers terminate prominently in the indusium griseum (Fig. 13E, G);
TH fibers do as well, but less densely. The indusium griseum (also known as the supracallosal gyrus) is situated between the cingulate gyrus and the corpus callosum. TH-positive fibers may be both dopaminergic and noradrenergic, with varying relative concentrations throughout the cortex (Williams and Goldman-Rakic, 1993).

\section{Summary of subcortical fibers}

Subcortical projections use all regions of the CB. However, amygdala fibers do not continue into caudal portions of the dorsal $\mathrm{CB}$, and AV/LD thalamus fibers do not extend into the subgenual CB. Subcortical fibers, including those from neuromodulator systems, use the $\mathrm{CB}$ to project to all cingulate regions, including sACC, dACC, PCC, and retrosplenial cortex. However, importantly, these subcortical fibers also use the $\mathrm{CB}$ to travel to other noncingulate cortical regions, including all of frontal and parietal dorsomedial cortex, as well as MTL. Amygdala and thalamic fibers do not appear to use the contralateral CB.

\section{CB topography}

Fibers from different brain regions, both cortical and subcortical, are mostly comingled within the CB. Overall, cortical, amygdala, and TH-positive fibers tend to occupy dorsal and medial positions within the dorsal $\mathrm{CB}$ (Figs. 5C, $7 B$, and $11 C)$. Prior observations of the corticocortical fibers within the CB have shown similar topography (Dejerine, 1895; Mufson and Pandya, 1984; Petrides and Pandya, 2007). However, in some coronal sections, cortical, amygdala, and $\mathrm{TH}-$ positive fibers appear to be evenly spread across the entire bundle (see particularly Figs. $8 B$ and $9 B$ ). Importantly, as fibers from any given region travel through the bundle, they shift positions. That is, at one point in the dorsal CB, a particular group of fibers may be mostly located in the dorsal portion of the bundle, but at another point, they may be located medially. For example, dACC fibers occupy a dorsal and medial position in the rostral portion of the dorsal $\mathrm{CB}$, then switch to occupying a more ventromedial zone a few millimeters caudally. Thus, the dorsal-ventral and medial-lateral topography of fibers within the CB is weak. AV/LD thalamus and SERT-positive fibers, in contrast, occupy a central portion of the bundle, lateral and ventral to the others (see also Mufson and Pandya, 1984) (Figs. $12 C$ and $13 E$ ).

\section{CB segmentation}

Based on the specific connections passing through this bundle, the CB can be segmented into four zones as follows: subgenual, rostral dorsal, caudal dorsal, and temporal (Fig. 14). Some fibers (serotonergic, dopaminergic/noradrenergic, AChE, PCC) travel through all four of these regions. The other fibers described in this study do not, and these restricted projections form the basis 
for $\mathrm{CB}$ segmentation. Importantly, these divisions are based not on gross bundle location and shape, but on projection systems. Figure 2 shows the projections through each segment of the CB from each injection site. Table 1 summarizes our results by brain region and lists the primary areas in which CB fibers terminate.

The subgenual $\mathrm{CB}$ does not contain significant numbers of dmFC, vlPFC, IOFC, and AV/LD thalamus fibers. Fibers that do use the subgenual $\mathrm{CB}$ fall into three categories: (1) sACC and OFC fibers that join the subgenual $\mathrm{CB}$ to reach dorsal targets or, in the case of sACC fibers, cross over the subgenual CB to reach other bundles, such as the uncinate fasciculus or corpus callosum; (2) dorsal prefrontal and cingulate cortical fibers (PCC, dACC, dlFC, FP) that terminate in sACC and mOFC; and (3) subcortical fibers (amygdala, SERT, TH, AChE) that use the subgenual CB both to terminate in sACC and to reach distant dorsal targets.

The rostral dorsal CB contains all of the frontal, subcortical, and cingulate fibers that use the CB. This section of the $\mathrm{CB}$ contains fibers traveling both anteriorly to rostral dACC, dmFC, and FP, and posteriorly to caudal dACC, dmFC, PCC, and MTL. It also contains fibers that project ventrally to the subgenual $\mathrm{CB}$. The caudal dorsal CB is similar but does not contain many amygdala, vlPFC, or lOFC fibers. The dividing line between these two segments is at approximately the anterior-posterior level of the rostral edge of the anterior commissure. For reference, in rhesus macaques, this is $\sim 20 \mathrm{~mm}$ anterior to the interaural plane.

The temporal CB contains PCC and subcortical (amygdala, dopaminergic/noradrenergic, serotonergic, AV/LD thalamic) fibers projecting to MTL. Dopaminergic/noradrenergic, AChE, and serotonergic fibers in the temporal $\mathrm{CB}$ also travel dorsally to reach the caudal dorsal CB. Frontal fibers (dACC, OFC, dlFC, dmFC, vlPFC, sACC, FP) do not travel significantly through the temporal CB.

\section{Neurotherapeutic target placement}

Cingulotomy and subcallosal DBS both target the CB. Based on our tract-tracing studies, we examined which fibers would most likely be affected by these neurosurgical interventions. To determine the fibers affected by cingulotomy procedures, we transformed the anterior-posterior range of possible cingulotomy lesions (Steele et al., 2008) to fit the monkey brain (see Materials and Methods). The cingulotomy lesion spans portions of both the rostral dorsal and caudal dorsal segments of the CB. Thus, it will ablate all of the fiber types investigated: substantial amounts of cingulate, noncingulate cortical, and subcortical fibers are all within the path of the lesion (Figs. 2 and 15A; Table 1). Significantly, the cingulotomy lesion is in one of the only CB locations that contains both AV/LD thalamic and amygdala fibers.

The electrode placement for subcallosal DBS for MDD (Fig. $15 B$ ) is in the caudal portion of the subgenual CB (Mayberg et al., 2005; Lozano et al., 2008), and thus will likely affect both subcortical and cortical fiber fibers in this bundle (Fig. 2; Table 1), along with ventral prefrontal cortical fibers in the nearby uncinate fasciculus (Lehman et al., 2011). Because all sACC fibers cross through the subgenual CB to reach distant bundles, stimulation will likely affect all sACC fibers originating in cell bodies near the stimulation site. OFC and SACC fibers traveling to the rostral dorsal $\mathrm{CB}$ via the subgenual $\mathrm{CB}$ will also be affected. Cortical fibers directly connecting with caudal sACC (from OFC, dlFC, cingulate, FP) will also be in the path of stimulation. However, cortical fibers projecting exclusively through the dorsal CB will be largely unaffected by DBS. Stimulation will also capture amygdala pathways as they travel to the rostral dorsal $\mathrm{CB}$ via the subgenual CB. Importantly, this target will not involve AV/LD thalamus, lOFC, vlPFC, or dmFC fibers, which are not present in large numbers in the subgenual CB.

\section{Discussion}

Using tract-tracing techniques, we have illustrated the route and composition of the $\mathrm{CB}$. This long association bundle courses from a subgenual position to a dorsal one, curving around the genu, continuing along a dorsal-medial route, then turning at the splenium to reach the medial temporal lobe (Fig. 4). Cingulate, noncingulate frontal, and subcortical fibers project through the $\mathrm{CB}$ to terminate in cingulate cortex. In addition, these axons use the $\mathrm{CB}$ to terminate in a number of areas outside of cingulate cortex (Table 1). Thus, we have demonstrated that many cortical and subcortical fibers that travel through the CB neither originate nor terminate in cingulate cortex. These connections have been alluded to previously (Goldman-Rakic et al., 1984; Mufson and Pandya, 1984; Morris et al., 1999), but the full extent of noncingulate pathways through the $\mathrm{CB}$ was not known. Their existence changes our concept of the CB: this bundle is not simply a pathway for cingulate fibers but represents a much broader connecting system. 
A
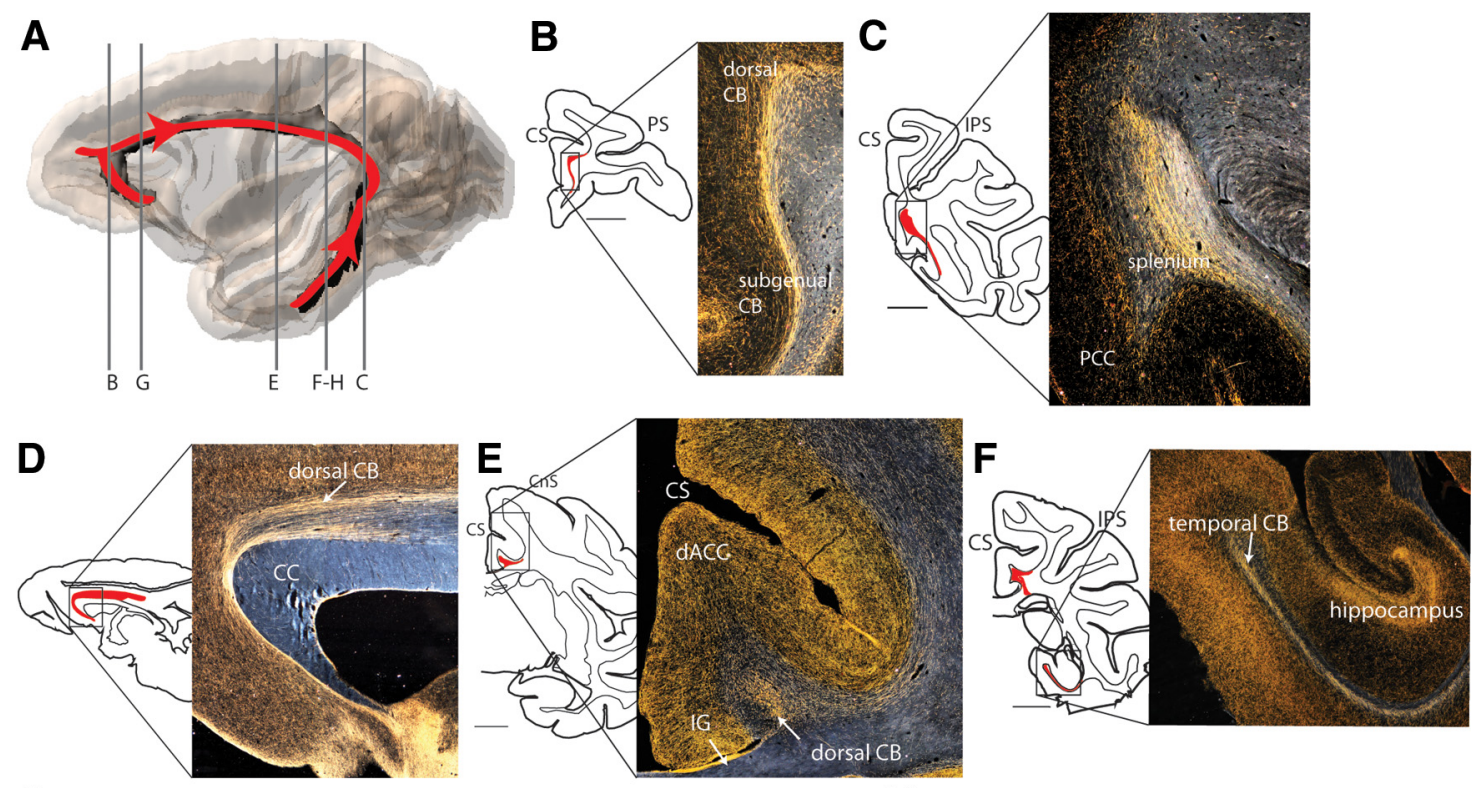

G
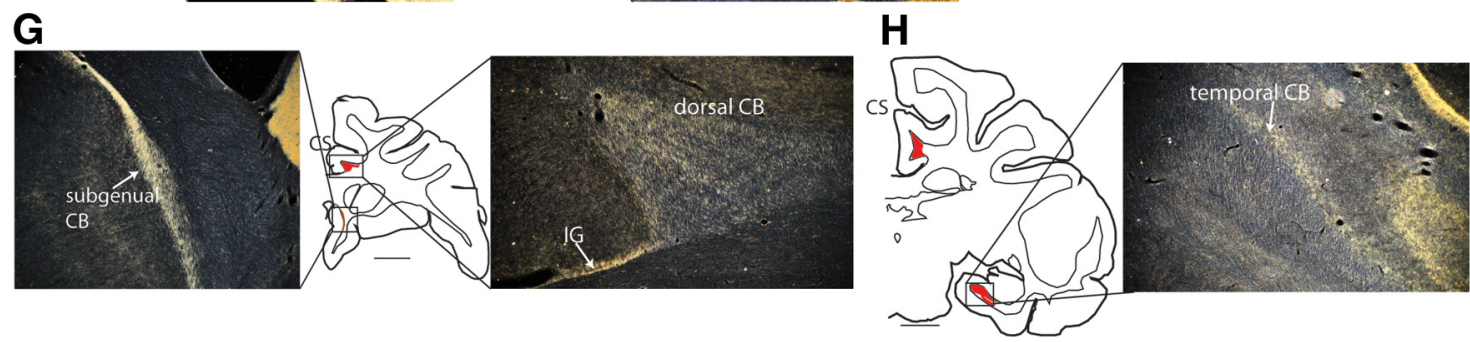

Figure 13. Dopaminergic/noradrenergic, serotonergic, and cholinergic fibers visualized with TH, SERT, and AChE staining, respectively, project through the CB. A, Schematic represents the extent of TH, SERT, and AChE projection through CB. Red represents TH/SERT/AChE projection. Black represents entirety of CB. $B$, TH fibers project through the subgenual and dorsal CB. C, TH fibers curve around the splenium, connecting the temporal $C B$ with the dorsal CB. D, A sagittal section represents SERT fibers connecting the uncinate fasciculus, subgenual $C B$, and dorsal CB. E, SERT fibers are present in the dorsal CB. $F$, SERT fibers are present in the temporal CB. G, AChE fibers project trough the subgenual (left) and dorsal (right) $C B$. $\boldsymbol{H}, A C h E$ fibers are present in the temporal CB. CC, Corpus callosum; CnS, central sulcus; CS, cingulate sulcus; IG, indusium griseum; IPS, intraparietal sulcus; PS, principal sulcus.

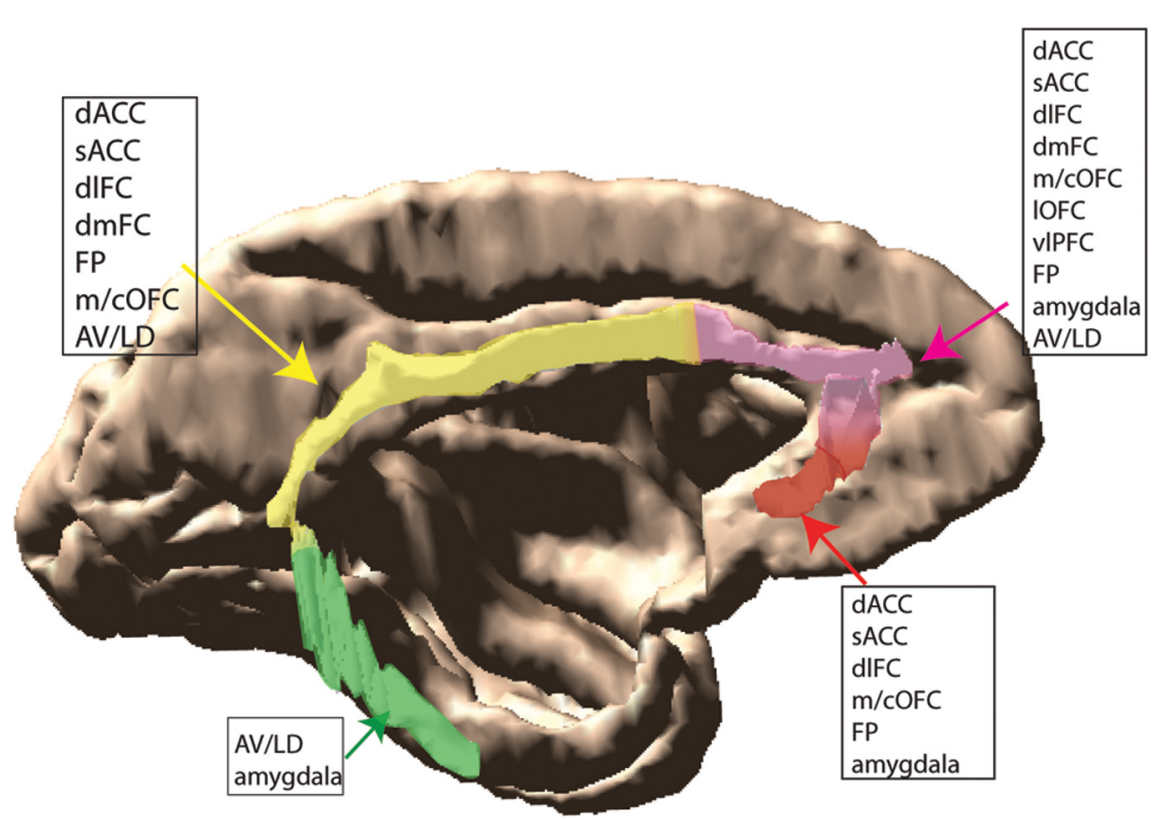

Figure 14. Segmentation of pathways through the subgenual (red), rostral dorsal (pink), caudal dorsal (yellow), and temporal (green) CB. Fibers that distinguish segments of the CB are listed in adjacent boxes. PCC, SERT, AChE, and TH fibers are present in all segments of the $C B$.
Because of its medial position within the brain and its relationship with MTL and cingulate cortex, prior investigations have emphasized the CB as one of the primary bundles of the limbic circuit (Yakovlev and Locke, 1961), containing both fibers from adjacent cortex continually entering and leaving the CB and long association axons projecting extensive distances within the bundle (Mettler, 1935; Yakovlev and Locke, 1961). The broadest investigation of the CB (Mufson and Pandya, 1984) identified three primary fiber components of the CB: thalamic, cingulate, and association cortex (frontal and posterior parietal). Goldman-Rakic et al. (1984) and Morris et al. (1999) demonstrated dlFC pathways through the CB, and Petrides and Pandya (2007) detailed the frontopolar projections through the CB. Our work builds on earlier findings, using numerous frontal, cingulate, and subcortical injections to both delineate the origin of fibers using the $\mathrm{CB}$ and to segment the bundle according to which fibers travel through each region. 
Table 1. Summary of fiber pathways through the CB, according to area ${ }^{a}$

\begin{tabular}{|c|c|c|c|c|c|}
\hline Area/stain & Subgenual CB & Rostral dorsal CB & Caudal dorsal CB & Temporal & Terminals from CB fibers \\
\hline SACC & + & + & + & $(-)$ & sACC, dACC, PCC, RSP, m0FC, FP, dmFC \\
\hline $\mathrm{dACC}$ & + & + & + & $(-)$ & SACC, dACC, PCC, RSP, 0FC, FP, dmFC, 7M \\
\hline $\mathrm{PCC}$ & + & + & + & + & sACC, dACC, PCC, RSP, dmFC, OFC, dIFC, 7M, MTL \\
\hline $\mathrm{m} / \mathrm{COFC}$ & + & + & + & $(-)$ & sACC, dACC, PCC, RSP, FP, dmFC, 7M \\
\hline IOFC & $(-)$ & + & $(-)$ & $(-)$ & $\mathrm{dACC}, \mathrm{dmFC}$ \\
\hline vIPFC & $(-)$ & + & $(-)$ & $(-)$ & $\mathrm{dACC}, \mathrm{dmFC}$ \\
\hline FP & + & + & + & - & sACC, dACC, PCC, RSP, dmFC \\
\hline $\mathrm{dlFC}$ & + & + & + & $(-)$ & sACC, dACC, PCC, RSP, FP, 7M, prosubiculum \\
\hline $\mathrm{dmFC}$ & $(-)$ & + & + & $(-)$ & $\mathrm{dACC}, \mathrm{PCC}, \mathrm{RSP}, 7 \mathrm{M}$ \\
\hline Amygdala & + & + & - & + & sACC, dACC, dmFC, MTL \\
\hline Thalamus & $(-)$ & + & + & + & $\mathrm{dACC}, \mathrm{PCC}, \mathrm{RSP}, \mathrm{dmFC}, 7 \mathrm{M}, \mathrm{MTL}$ \\
\hline SERT & + & + & + & + & sACC, dACC, PCC, RSP, dmFC, 7M, IG, MTL, caudal dmC \\
\hline TH & + & + & + & + & sACC, dACC, PCC, RSP, dmFC, 7M, IG, MTL, caudal dmC \\
\hline AChE & + & + & + & + & sACC, dACC, PCC, RSP, dmFC, 7M, IG, MTL, caudal dmC \\
\hline
\end{tabular}

${ }^{a}$ Data represent the strongest fiber projection observed across cases for a given area. + , bundle present; $(-$ ), scattered fibers present; - , fibers absent; $7 \mathrm{M}$, parietal area $7 \mathrm{M}$ (precuneus); dmC, dorsomedial cortex; IG, indusium griseum; RSP, retrosplenial cortex.

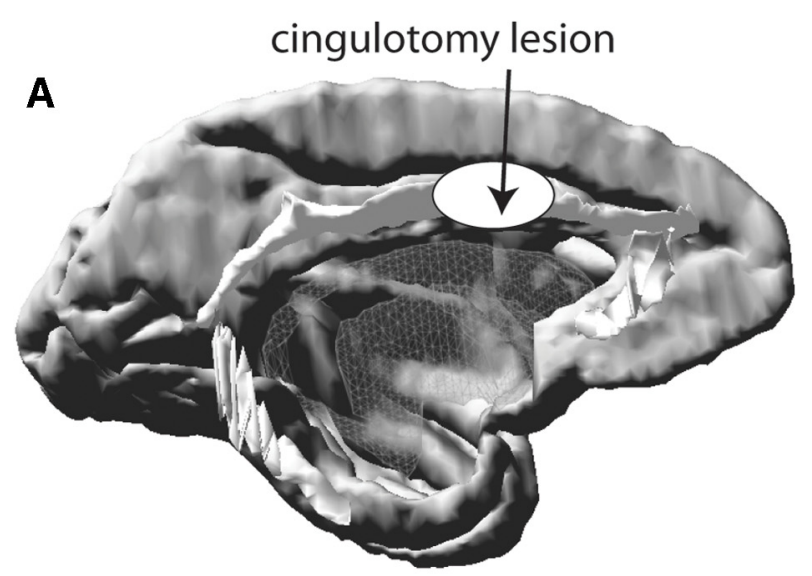

B

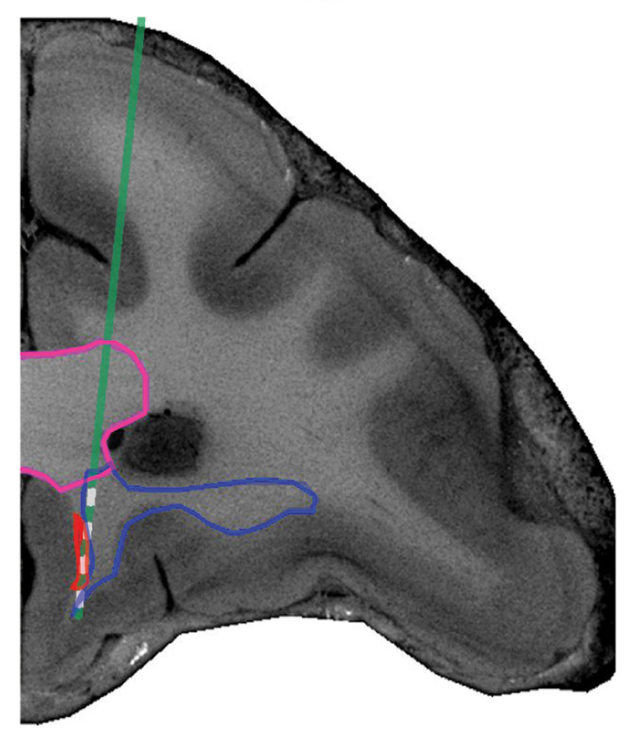

Figure 15. Location of neurosurgical interventions for MDD and OCD affecting the CB. $A$, The cingulotomy lesion is located in the dorsal $C B$ and $\mathrm{dACC}$. $\boldsymbol{B}$, The electrode (green; contact points in white) for subcallosal DBS for MDD intersects the subgenual $C B$ (red), uncinate fasciculus (blue), and corpus callosum (pink).

Prior literature was confusing on the topic of the subgenual CB. Although early work did identify a subgenual CB component (Yakovlev and Locke, 1961), more recent descriptions of white matter pathways have ignored this part of the bundle. Mufson and Pandya (1984) did not include the subgenual component in their description of the CB, although they showed fibers traveling from a dorsal to ventral position. Schmahmann and Pandya (2006) took a similar view, acknowledging the existence of (some of) the fibers we mention, but assigning them instead to the uncinate fasciculus. However, some neuroimagers and DBS groups have been using the term "subgenual cingulum bundle" (Kim et al., 2006) or "subgenual cingulate white matter" (Johansen-Berg et al., 2008). Without a clear neuroanatomical underpinning, it is difficult to interpret which connections these might involve. Our work, at least in part, resolves these issues.

\section{Segmenting the CB}

Based on connections traveling through different regions of the $\mathrm{CB}$, we segmented the bundle into four distinct portions: rostral subgenual; rostral dorsal; caudal dorsal; and temporal (Fig. 14). Many fibers travel through all four segments (Table 1). Fibers from the frontal cortex, thalamus, and amygdala form the basis for segmenting the bundle. The subgenual $\mathrm{CB}$ contains not only axons projecting to and from the sACC, but also fibers that use the subgenual $\mathrm{CB}$ to reach the dorsal $\mathrm{CB}$. $\mathrm{AV} / \mathrm{LD}$ thalamus, dmFC, vlPFC, and IOFC fibers are largely absent from the subgenual $\mathrm{CB}$. In contrast, the rostral dorsal $\mathrm{CB}$ contains fibers from all regions we examined here. These axons travel rostrally and caudally to reach dACC, FP, dmFC, and the caudal dorsal and subgenual $\mathrm{CB}$. The caudal dorsal $\mathrm{CB}$ is similar to the rostral dorsal $\mathrm{CB}$, with three important exceptions: few amygdala, vlPFC, or lOFC fibers use this part of the CB. Finally, the temporal CB contains subcortical and PCC (but few frontal) fibers terminating in MTL or connecting with the caudal dorsal CB. We have not included temporal, parietal, or retrosplenial cortical projections through the $\mathrm{CB}$ here; in the future, these may be used to segment the bundle further, particularly in the temporal zone. Furthermore, our segmentation depends on not finding fibers in particular locations. Although we have reduced the risk of a false negative by including several cases for each region, we cannot rule out the possibility that some fibers from a given region do pass through a segment. In particular, dlFC may project more rostrally in the temporal CB than we have identified, in accordance with prior literature (Goldman-Rakic et al., 1984).

\section{Studies of human connectivity}

Diffusion magnetic resonance imaging (dMRI) is used to examine white matter connectivity in humans. Our results can guide 
interpretation of these data. Previously, we showed the organization of ventral prefrontal cortical axons in monkeys and found that these rules could be replicated using dMRI in monkeys and humans (Lehman et al., 2011; Jbabdi et al., 2013). Here, we have identified fiber pathways through the CB. Furthermore, we note several instances in which small numbers of scattered fibers travel through the CB in the monkey. Such pathways may be enhanced in the human because of species differences, although the trajectory may remain unchanged.

Differences between CB segments can highlight which connections are likely to be abnormal in dMRI studies of psychiatric disorders. For example, fractional anisotropy is reduced in the equivalent of the rostral dorsal $\mathrm{CB}$, but not the caudal dorsal $\mathrm{CB}$, of patients with bipolar disorder (Wang et al., 2008). Our results point to specific connections that may underlie this pattern. Fractional anisotropy differences between the rostral dorsal and caudal dorsal $\mathrm{CB}$ may be due to abnormalities in the amygdala's connections with dACC and dmFC. Consistent with this hypothesis, bipolar patients show abnormal amygdala-ACC functional connectivity (Anand et al., 2009). Rostral dorsal CB disruption may also reflect aberrations in the OFC connections with dACC or $\mathrm{dmFC}$, consistent with the putative role of OFC in reward deficits in bipolar disorder (Cotter et al., 2005). Finally, this abnormality may reflect pathology in dACC axons crossing over the $\mathrm{CB}$ to reach subcortical, lateral, and contralateral regions. An abnormality specific to the rostral dorsal CB is unlikely to reflect changes in connections with PCC or precuneus. It would be of interest to determine whether the subgenual $\mathrm{CB}$ is altered in these patients. If the abnormality reflects an underlying pathology in amygdala or ventral cortical connections with dACC or dmFC, we expect the subgenual $\mathrm{CB}$ to also be abnormal. If it instead reflects dysregulated dACC-subcortical connections, we would expect the subgenual CB to be relatively normal. In this way, our segmentation can be leveraged to identify the connections that underlie white matter abnormalities.

\section{Neurosurgical interventions for psychiatric disorders}

Neurosurgical interventions for treatment-resistant OCD and MDD target specific CB segments. The DBS electrode for MDD contains four contact points, each of which may affect a unique set of fibers (Mayberg et al., 2005). The angled electrode trajectory causes contact points to vary on both the dorsal-ventral and medial-lateral axes. There is also some electrode placement variation on every axis from patient to patient (Hamani et al., 2009). Dorsal contact points may intercept the corpus callosum (and thus affect ventral prefrontal contralateral projections); lateral contacts may intercept fibers in the uncinate fasciculus (and thus affect widespread OFC connections); finally, medial contacts likely intercept the subgenual $\mathrm{CB}$. Through the subgenual $\mathrm{CB}$, DBS likely affects sACC cortical, lateral, and subcortical connections. However, the subgenual CB is also a major route by which ventral prefrontal cortical and subcortical fibers access the dorsal $\mathrm{CB}$. As such, it may capture fibers from areas distant to the electrode, including OFC and amygdala, as they travel to dACC, dmFC, PCC, and precuneus. A recent dMRI case study showed that the therapeutically effective electrode contact intercepted subgenual CB fibers extending dorsally (Lujan et al., 2013). In linking together affective, self-reflective, and cognitive control systems, the dACC and $\mathrm{dmFC}$ are central to MDD pathology (Sheline et al., 2010). DBS efficacy may depend on targeting this dorsal system via subgenual CB fibers.

The cingulotomy lesion, by contrast, is located at the intersection of the rostral dorsal and caudal dorsal CB segments (for both
OCD and MDD). The cingulotomy will likely affect nearby dACC fibers crossing the dorsal CB to reach lateral, contralateral, and subcortical targets. It is also in a position to interrupt widespread connections. Fibers in this area travel anteriorly and posteriorly, connecting amygdala, thalamus, prefrontal cortices, and neurotransmitter systems with cingulate, $\mathrm{dmFC}$, and precuneus. Intriguingly, more rostral cingulotomy lesions may be more effective at treating MDD (Steele et al., 2008). A major difference between the rostral and caudal portions of the $\mathrm{CB}$ is the presence of amygdala, vlPFC, and IOFC fibers. Established MDD abnormalities in these areas, particularly the amygdala (Hastings et al., 2004; Siegle et al., 2007), suggest that the effectiveness of the lesion may depend on capturing amygdala fibers. In summary, we have identified the specific connections impacted by cingulotomy and DBS. This basic knowledge is fundamental both to understanding neurosurgical interventions for MDD and OCD and to delineating the circuit-level changes associated with psychiatric disorders more broadly.

\section{References}

Anand A, Li Y, Wang Y, Lowe MJ, Dzemidzic M (2009) Resting state corticolimbic connectivity abnormalities in unmedicated bipolar disorder and unipolar depression. Psychiatry Res 171:189-198. CrossRef Medline

Baer L, Rauch SL, Ballantine HT Jr, Martuza R, Cosgrove R, Cassem E, Giriunas I, Manzo PA, Dimino C, Jenike MA (1995) Cingulotomy for intractable obsessive-compulsive disorder: prospective long-term follow-up of $18 \mathrm{pa}-$ tients. Arch Gen Psychiatry 52:384-392. CrossRef Medline

Bevore C (1891) On the course of the fibers of the cingulum and posterior parts of the corpus callosum and fornix in the Marmoset monkey. Philos Trans 182:135-200.

Cotter D, Hudson L, Landau S (2005) Evidence for orbitofrontal pathology in bipolar disorder and major depression, but not in schizophrenia. Bipolar Disord 7:358-369. CrossRef Medline

Croxson PL, Johansen-Berg H, Behrens TE, Robson MD, Pinsk MA, Gross CG, Richter W, Richter MC, Kastner S, Rushworth MF (2005) Quantitative investigation of connections of the prefrontal cortex in the human and macaque using probabilistic diffusion tractography. J Neurosci 25: 8854-8866. CrossRef Medline

Dejerine J (1895) Anatomie des centres nerveux. Paris: Rueff.

Geneser-Jensen FA, Blackstad TW (1971) Distribution of acetyl cholinesterase in the hippocampal region of the guinea pig. Z Zellforsch 114:460 481. CrossRef Medline

Goldman-Rakic PS, Selemon LD, Schwartz ML (1984) Dual pathways connecting the dorsolateral prefronal cortex with the hippcampal formation and parahippocampal cortex in the rhesus monkey. Neuroscience 12: 719-743. CrossRef Medline

Greenberg BD, Rauch SL, Haber SN (2010) Invasive circuitry-based neurotherapeutics: stereotactic ablation and deep brain stimulation for OCD. Neuropsychopharmacology 35:317-336. CrossRef Medline

Haber SN, Lynd-Balta E, Mitchell SJ (1993) The organization of the descending ventral pallidal projections in the monkey. J Comp Neurol 329: 111-128. CrossRef Medline

Haber SN, Kim KS, Mailly P, Calzavara R (2006) Reward-related cortical inputs define a large striatal region in primates that interface with associative cortical inputs, providing a substrate for incentive-based learning. J Neurosci 26:8368-8376. CrossRef Medline

Hamani C, Mayberg H, Snyder B, Giacobbe P, Kennedy S, Lozano AM (2009) Deep brain stimulation of the subcallosal cingulate gyrus for depression: anatomical location of active contacts in clinical responders and a suggested guideline for targeting. J Neurosurg 111:1209-1215. CrossRef Medline

Hastings RS, Parsey RV, Oquendo MA, Arango V, Mann JJ (2004) Volumetric analysis of the prefrontal cortex, amygdala, and hippocampus in major depression. Neuropsychopharmacology 29:952-959. CrossRef Medline

Jbabdi S, Lehman JF, Haber SN, Behrens TE (2013) Human and monkey ventral prefrontal fibers use the same organizational principles to reach their targets: tracing versus tractography. J Neurosci 33:3190-3201. CrossRef Medline

Johansen-Berg H, Gutman DA, Behrens TE, Matthews PM, Rushworth MF, 
Katz E, Lozano AM, Mayberg HS (2008) Anatomical connectivity of the subgenual cingulate region targeted with deep brain stimulation for treatment-resistant depression. Cereb Cortex 18:1374-1383. CrossRef Medline

Kappers C, Huber G, Crosby E (1936) The comparative anatomy of the nervous system of vertebrates including man. New York: Macmillan.

Keedwell PA, Chapman R, Christiansen K, Richardson H, Evans J, Jones DK (2012) Cingulum white matter in young women at risk of depression: the effect of family history and anhedonia. Biol Psychiatry 72:296-302. CrossRef Medline

Kim SJ, Jeong DU, Sim ME, Bae SC, Chung A, Kim MJ, Chang KH, Ryu J, Renshaw PF, Lyoo IK (2006) Asymmetrically altered integrity of cingulum bundle in posttraumatic stress disorder. Neuropsychobiology 54: 120-125. CrossRef Medline

Lehman JF, Greenberg BD, McIntyre CC, Rasmussen SA, Haber SN (2011) Rules ventral prefrontal cortical axons use to reach their targets: implications for diffusion tensor imaging tractography and deep brain stimulation for psychiatric illness. J Neurosci 31:10392-10402. CrossRef Medline

Lozano AM, Mayberg HS, Giacobbe P, Hamani C, Craddock RC, Kennedy SH (2008) Subcallosal cingulate gyrus deep brain stimulation for treatmentresistant depression. Biol Psychiatry 64:461-467. CrossRef Medline

Lujan JL, Chaturvedi A, Choi KS, Holtzheimer PE, Gross RE, Mayberg HS, McIntyre CC (2013) Tractography-activation models applied to subcallosal cingulate deep brain stimulation. Brain Stimul 6:737-739. CrossRef Medline

Mayberg HS, Lozano AM, Voon V, McNeely HE, Seminowicz D, Hamani C, Schwalb JM, Kennedy SH (2005) Deep brain stimulation for treatmentresistant depression. Neuron 45:651-660. CrossRef Medline

Mettler R (1935) Corticofugal fiber connections of the cortex of Macaca mulatta: the frontal region. J Comp Neurol 61:509-542. CrossRef

Morris R, Pandya DN, Petrides M (1999) Fiber system linking the middorsolateral frontal cortex with the retrosplenial/presubicular region in the rhesus monkey. J Comp Neurol 407:183-192. CrossRef Medline

Mufson EJ, Pandya DN (1984) Some observations on the course and composition of the cingulum bundle in the rhesus monkey. J Comp Neurol 225:31-43. CrossRef Medline
Petrides M, Pandya DN (2007) Efferent association pathways from the rostral prefrontal cortex in the macaque monkey. J Neurosci 27:1157311586. CrossRef Medline

Schmahmann J, Pandya D (2006) Fiber pathways of the brain. New York: Oxford UP.

Sheline YI, Price JL, Yan Z, Mintun MA (2010) Resting-state functional MRI in depression unmasks increased connectivity between networks via the dorsal nexus. Proc Natl Acad Sci U S A 107:11020-11025. CrossRef Medline

Siegle GJ, Thompson W, Carter CS, Steinhauer SR, Thase ME (2007) Increased amygdala and decreased dorsolateral prefrontal BOLD responses in unipolar depression: related and independent features. Biol Psychiatry 61:198-209. CrossRef Medline

Steele JD, Christmas D, Eljamel MS, Matthews K (2008) Anterior cingulotomy for major depression: clinical outcome and relationship to lesion characteristics. Biol Psychiatry 63:670-677. CrossRef Medline

Szeszko PR, Ardekani BA, Ashtari M, Malhotra AK, Robinson DG, Bilder RM, Lim KO (2005) White matter abnormalities in obsessivecompulsive disorder: a diffusion tensor imaging study. Arch Gen Psychiatry 62:782-790. CrossRef Medline

Wang F, Jackowski M, Kalmar JH, Chepenik LG, Tie K, Qiu M, Gong G, Pittman BP, Jones MM, Shah MP, Spencer L, Papademetris X, Constable RT, Blumberg HP (2008) Abnormal anterior cingulum integrity in bipolar disorder determined through diffusion tensor imaging. Br J Psychiatry 193:126-129. CrossRef Medline

Williams SM, Goldman-Rakic PS (1993) Characterization of the dopaminergic innervation of the primate frontal cortex using a dopaminespecific antibody. Cereb Cortex 3:199-222. CrossRef Medline

Yakovlev PI, Locke S (1961) Limbic nuclei of the thalamus and connections of limbic cortex: III. Corticocortical connections of the anterior cingulate gyrus, the cingulum, and the ubcallosal bundle in monkey. Arch Neurol 5:364-400. CrossRef Medline

Yin D, Valles FE, Fiandaca MS, Forsayeth J, Larson P, Starr P, Bankiewicz KS (2009) Striatal volume differences between nonhuman and human primates. J Neurosci Methods 176:200-205. CrossRef Medline 